\title{
Polysaccharide-based Nanoparticles for Theranostic Nanomedicine
}

Special Issue: Non-Antigenic Regulators of Targeting for Imaging and Therapy

M. Swierczewska ${ }^{1}$, H. S. $\operatorname{Han}^{2}$, K. Kim ${ }^{3}$, J.H. Park ${ }^{2 *}$, S. Lee ${ }^{1 *}$

${ }^{1}$ Russell H. Morgan Department of Radiology and Radiological Science, Center for Cancer Nanotechnology Excellence, Center for Nanomedicine at the Wilmer Eye Institute, Johns Hopkins University, 400 North Broadway, Baltimore, MD 21231 (USA)

${ }^{2}$ School of Chemical Engineering, College of Engineering, Sungkyunkwan University, Suwon 440-746, Republic of Korea

${ }^{3}$ Center for Theragnosis, Biomedical Research Institute, Korea Institute of Science and Technology, Seoul 136-791, Republic of Korea

*Corresponding Authors

Keywords: Nanoparticles, Drug Delivery, Carbohydrate, Hyaluronan, Chitosan, Molecular Imaging 


\begin{abstract}
Polysaccharides are natural biological molecules that have numerous advantages for theranostics, the integrated approach of therapeutics and diagnostics. Their derivable reactive groups can be leveraged for functionalization with a nanoparticle-enabling conjugate, therapeutics (small molecules, proteins, peptides, photosensitizers) and/or diagnostic agents (imaging agents, sensors). In addition, polysaccharides are diverse in size and charge, biodegradable and abundant and show low toxicity in vivo. Polysaccharide-based nanoparticles are increasingly being used as platforms for simultaneous drug delivery and imaging and are therefore becoming popular theranostic nanoparticles. The review focuses on the method of nanoparticle formation (selfassembled, physical or chemical cross-linked) when engineering polysaccharide-based nanoparticles for theranostic nanomedicine. We highlight recent examples of polysaccharidesbased theranostic systems from literature and their potential for use in the clinic, particularly chitosan- and hyaluronic acid-based NPs.
\end{abstract}




\section{$\underline{\text { Table of Contents }}$}

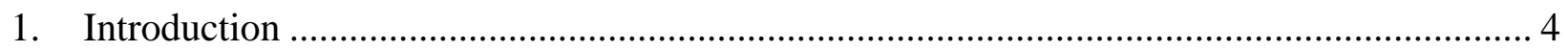

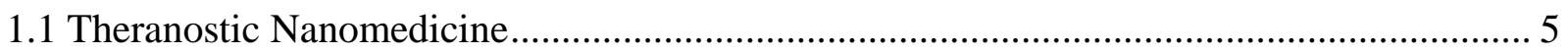

1.2 Design Considerations of Polysaccharide-based Theranostic Nanoparticles ..................... 8

2. Polysaccharide Modification for Theranostics ............................................................. 9

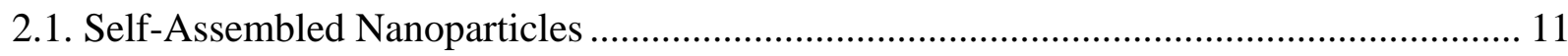

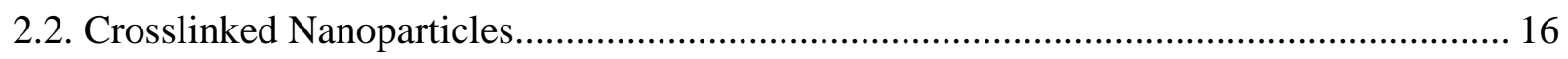

2.2.1. Ionically Crosslinked Nanoparticles................................................................... 16

2.2.2. Covalently Crosslinked Nanoparticles ............................................................. 17

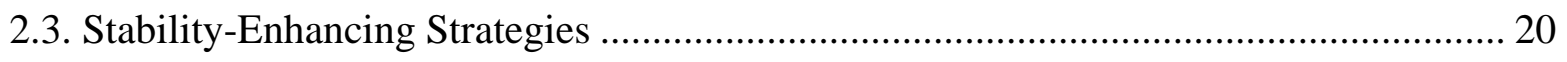

3. Application of Polysaccharide-Based Nanoparticles for Theranostic Nanomedicine ............. 21

3.1. Hyaluronic acid-based Theranostic Nanoparticles.................................................... 25

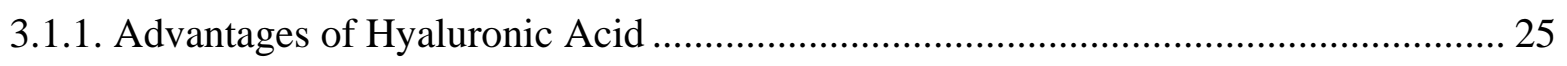

3.1.2. Application of Hyaluronic Acid-Based NPs ........................................................... 26

3.1.3. Future of Hyaluronic Acid-Based Nanoparticles for Theranostics ......................... 29

3.2. Chitosan-based Theranostic Nanoparticles ............................................................... 29

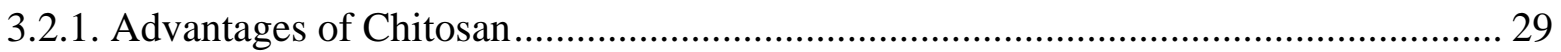

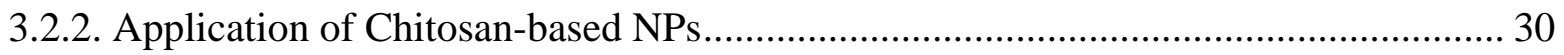

3.2.3. Future of Chitosan-Based Nanoparticles for Theranostics .................................... 34

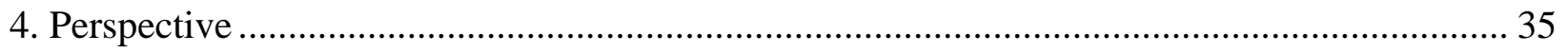

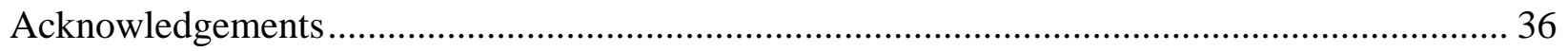

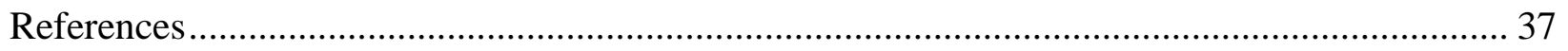




\section{Introduction}

Carbohydrates, $\left(\mathrm{CH}_{2} \mathrm{O}\right)_{n}$, are a vast class of molecules found abundantly in nature that encompass simple sugars up to large polysaccharides. Monosaccharides $(n=3,5,6)$, the simplest of carbohydrates, are the building blocks of all other carbohydrates. Most carbohydrates in nature are polysaccharides, which form through a series of condensation reactions among monosaccharides. With a wide array of simple sugars, their isomers, and assorted linkages via hydroxyl groups, carbohydrates are quite diverse molecules. Their derivable reactive groups give rise to many different products ranging in molecular weight, structure and charge. Because of their diversity, carbohydrates are highly information-rich molecules in the body and have many critical biological functions. They serve as a source of cellular energy, the building blocks of nucleic acids, structural components in the cells, and have key roles in cell-cell recognition and adhesion, among other biological processes. Carbohydrates are highly stable, non-toxic, hydrophilic and biodegradable in the human body. In their natural state, many carbohydrates have hydroxyl, carboxyl and amino groups that can interact with biological tissue in a noncovalent manner and are therefore considered bioadhesive, particularly to mucosal surfaces, allowing the material to have prolonged half-life in the body. Taken together, the characteristics of carbohydrates meet the requirements of an effective platform for in vivo drug delivery and imaging. With advancements in polymer science, carbohydrates can be engineered as biomedical tools with unique structures and defined functions. Carbohydrates, predominantly polysaccharides, have been incorporated into nanoparticles (NPs) and investigated in hydrogels and nanofibers for over two decades $[1,2]$. In this review, we will focus on recent polysaccharide-based NPs, particularly NPs consisting of chitosan and hyaluronic acid, in drug delivery and imaging applications. In this section, we will introduce the goals of theranostic 
nanomedicine, the advantages of carbohydrate NPs (1.1), and the design criteria for polysaccharide-based theranostic nanomedicines (1.2).

\subsection{Theranostic Nanomedicine}

Theranostics is the integration of therapeutics and diagnostics, which can be administered simultaneously or sequentially. NPs are the key to the application of nanotechnology in medicine, defined as nanomedicine, and are effective in multifunctionality for theranostic strategies. The goal of theranostic nanomedicine is to tailor therapies to a patient's specific diagnosis and improve treatment outcomes with fewer side effects in a shorter amount of time than current trial-and-error treatment regimens. NPs have significant advantages in theranostics because of their targetability and multifunctionality [3]. By passive and/or active targeting, NPs can target the disease site. Once there, theranostic NPs may diagnose the disease by reporting on the location of the disease, the stage of disease or the response to treatment [4]. Finally, NPs can deliver a therapeutic agent to the targeted site at necessary concentrations, potentially in response to molecular signals or external stimuli. Overall, theranostic nanomedicine can be used to monitor drug delivery, drug release and drug efficacy. In this review, we show that polysaccharide-based NPs are especially suited for theranostic nanomedicine.

NPs have significant advantages to carry drugs and other cargo in the body. First, they protect the load from premature chemical or enzymatic degradation. Secondly, because of their high surface to volume ratios, NPs can transport a large amount of cargo with less materials. Depending on the particle design, they can efficiently hold diverse cargo in the particle core and/or on the exterior surface by conjugation or adsorption. Because of this efficient loading capability, NPs decorated with ligands have shown improved cellular targeting and potency by multivalent binding [5, 6], depending on the NP size [7]. Unlike small molecules, NPs can 
simultaneously deliver diverse cargos to the target site [8]. Most importantly, NPs can passively target disease sites depending on their size, shape and charge [9]. They can pass through the smallest capillary vessels and penetrate cells to arrive at the target site. NPs are particularly suited for cancer applications because they can collect in certain solid tumors $[10,11]$ through the hyper-permeable (so-called 'leaky') vasculature that solid tumors exhibit, and the particles can remain at the site because of the tumor's poor lymphatic drainage; collectively, this effect is called the enhanced permeability and retention (EPR) effect $[12,13]$. Based on some animal studies, the EPR effect can lead to nearly 50 times greater accumulation in cancer models than healthy animal models [14] and is enhanced with an increase in the NP circulation half-life. The targeting ability of drug-loaded NPs via EPR prevents potentially severe adverse effects to healthy tissue caused by systemically-delivered active drugs. Additionally, NPs are tunable in size and act as modifiable platforms for targeting agents, enabling customizable biodistribution profiles in vivo. The ideal theranostic nanomedicine selectively and rapidly accumulates at its intended target, emits a signal based on a specific biomarker, and delivers an appropriate therapy based on the result. NPs should be non-toxic and degrade in the body without any harmful byproducts [9]. There are several excellent reviews on nanomedicine and NPs in drug delivery and molecular imaging [14-18]. With advancements in chemical modification and nanotechnology, polysaccharide NPs; can be synthesized with tunable shapes and sizes for therapeutic and imaging applications [19-21]. A number of recent reviews on polysaccharidebased NPs provide a comprehensive account on their advantages in biomedical applications [2225]. In this review, we will highlight recent polysaccharide-based NPs and their emergence as theranostic tools. 
A representative approach that exemplifies the benefits of theranostics is image-guided photodynamic therapy (PDT). PDT is a clinically approved phototherapy for cancer and other diseases. It requires activating a photosensitizing agent in the body by a tuned external wavelength in the presence of oxygen to induce cytotoxic reactive oxygen species at the localized area of the agent [26]. NPs for image-guided PDT can direct non-toxic photosensitizing agents and possibly other imaging probes to the site of disease, guiding clinician decisions to where external laser irradiation should be applied. If fluorescence imaging is used, photobleaching during laser irradiation can signify how much of the agent is being irradiated and can be used as a form of dosimetry in research and the clinic. Near-infrared fluorescence imaging is used in biomedical research in animal models, but is limited for clinical use because blood and other endogenous substances limit light penetration into tissue [27]. Other clinically relevant types of imaging contrast agents, like Gd for MRI or radiolabels for PET/SPECT imaging, can be functionalized to NPs to guide treatment. Finally, after laser irradiation, the photosensitizing agent can produce cytotoxic reactive oxygen species and lead to localized cell death and tissue destruction. In summary, the NP for image-guide PDT serves as a theranostic agent because it acts as an imaging probe and photosensitizing agent. Image-guided PDT demonstrates how a combined imaging and treatment strategy can fine-tune clinically approved therapies and provide more information about the treatment efficacy than separate imaging and treatment methods. Recently, polysaccharide-based theranostic NPs coupled with photosensitizers like Chlorin e6 (Ce6) or gold nanoparticles have been applied for image-guided PDT and other phototherapies $[26,28-32]$.

As seen by the example of image-guided PDT, theranostic nanomedicine holds great potential in therapy. Theranostics may speed up drug development by enabling treatment 
response monitoring in preclinical models and/or during clinical trials for patient stratification. Drug delivery and mechanism of action can be imaged in vivo in real-time using molecular imaging [33] to indicate when the drug is released from the nanocarrier and when specific molecular responses are reached as a cause of treatment. For example, response to anticancer drug therapy can be monitored by imaging cancer cell death through detection of apoptotic markers non-invasively in real-time to efficiently develop new therapeutic regimens. As a result, pharmacokinetics and pharmacodynamics of the active drug can be determined in a more rapid and non-invasive manner. Theranostic nanomedicine can serve as valuable preclinical and clinical research tools to enable personalized medicine.

\subsection{Design Considerations of Polysaccharide-based Theranostic Nanoparticles}

Polysaccharide-based NPs have the following advantages in theranostic nanomedicine. Firstly, using a natural biomaterial like polysaccharides to prepare NPs as opposed to metals or other synthetic polymers, minimizes concerns over toxicity, biodegradability, and physiological stability. Secondly, studies show that polysaccharide-based NPs may decrease uptake by the mononuclear phagocyte system [34] over other types of NPs [35], which prolongs the NP in vivo residence time and increases the possibility of disease site accumulation. Residence time of polysaccharide-based materials are also extended due to the bioadhesion of polysaccharides, particularly to mucosal surfaces. Also, carbohydrates are abundant in nature, making them costeffective biomaterials to investigate. Finally, most polysaccharide-based NPs hold derivable groups that can be used to conjugate targeting, therapeutic and imaging agents, making them diverse multifunctional biomedical tools for theranostic nanomedicine. Recent examples of polysaccharide-based NPs with theranostic functionality are highlighted in Section 3. 
When engineering polysaccharide-based NPs for theranostic nanomedicine, the route of administration, retention time and type of imaging and therapy must be considered. These factors may depend on the type of polysaccharide chosen, its key characteristics, size, and structure. For example, the charge of the polysaccharide is critical when formulating NPs, as discussed in Section 2. Chitosan is positively charged so low molecular weight polyanions can serve as ionic crosslinkers, while other types of polysaccharides are negatively charged, including heparin, alginate and hyaluronic acid (HA). In addition to charge, the unique biological function of the polysaccharide may direct the development of polysaccharide-based theranostics NPs. For example, HA intrinsically targets CD44 receptors; therefore, the glycosaminoglycan can serve as a targeting moiety for cancer therapy because various tumor cells are known to overexpress CD44 [36-39]. Heparin is a sulfated polysaccharide approved as an injectable anticoagulant drug that binds and inhibits the protein thrombin [40]. Studies show that heparin has anti-angiogenic and anti-cancer abilities and that these properties can be retained in heparin NPs [41, 42]. Overall, polysaccharide properties can synergize with the benefits of NPs to develop advanced theranostic nanomedicine.

In this review, we will introduce how NPs can be prepared using carbohydrates, namely polysaccharides (Section 2). Then, we will highlight examples of polysaccharide-based theranostic systems, particularly based on hyaluronic acid or chitosan, reported since 2010 (Section 3). Finally, we will provide a prospective into the future of polysaccharide-based nanoparticles, particularly in regards to clinical translation (Section 4).

\section{Polysaccharide Modification for Theranostics}

Owing to the presence of diverse functional groups, polysaccharides have been readily modified for biomedical applications such as drug delivery systems, tissue engineering, and 
regenerative medicine [19, 43-45]. For theranostics, both therapeutic and imaging agents can be covalently conjugated at the backbone of polysaccharides or physically encapsulated into the polysaccharides-based nanoparticles. The widely used functional groups in polysaccharides available for chemical modification, include hydroxyl, amino, aldehyde, and carboxylic acid groups [46-49]. The hydroxyl group is useful to react with compounds bearing anhydrides, carboxylic acids, or epoxides. Chemical modification is usually performed with the primary hydroxyl group at C6 of the sugar (e.g., dextran, chitosan, and hyaluronic acid) due to the higher reactivity of this position relative to secondary or tertiary moieties. The carboxylic acid and amino groups are frequently reacted by forming the amide bond via carbodiimide-mediated reactions. Polysaccharides with reducing ends allows for reductive amination in the presence of sodium cyanoborohydride. While other functional groups are found at the repeating unit of polysaccharides, the aldehyde group is only available at the reducing chain end, thus allowing for selective modification. The detailed chemistry for carbohydrate modification has been published elsewhere $[21,50,51]$. In this section, we focus on strategies for polysaccharide modification to prepare nano-sized particles which have potential for theranostics. The representative strategies include: preparing amphiphilic polysaccharides that can self-assemble into NPs in aqueous conditions, forming polyelectrolyte complexes (PECs) with oppositely charged carbohydrates, and introducing crosslinkers which enable formation of stable carbohydrate NPs (Figure 1). 


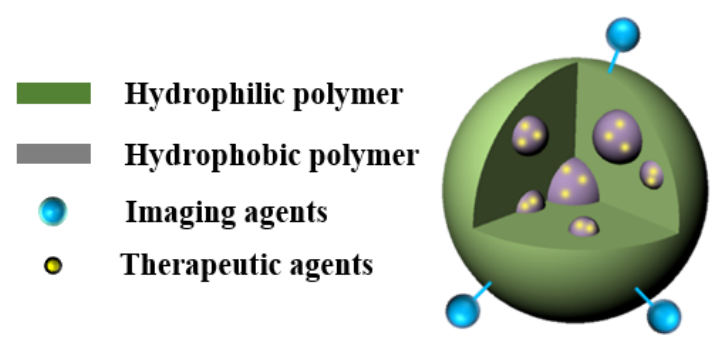

Self-assembled nanoparticles

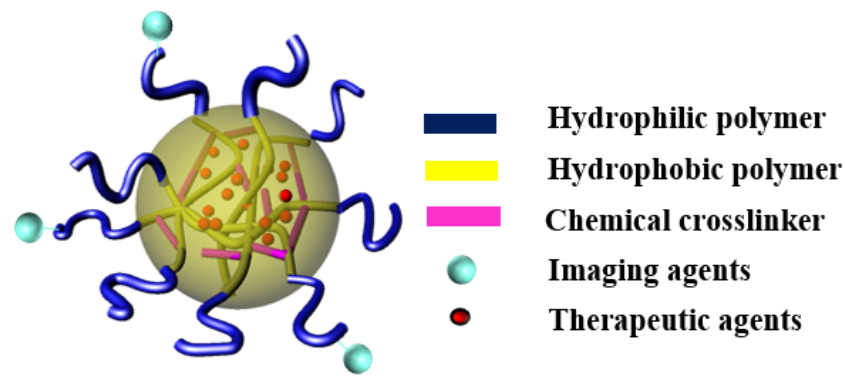

Chemically crosslinked nanoparticles

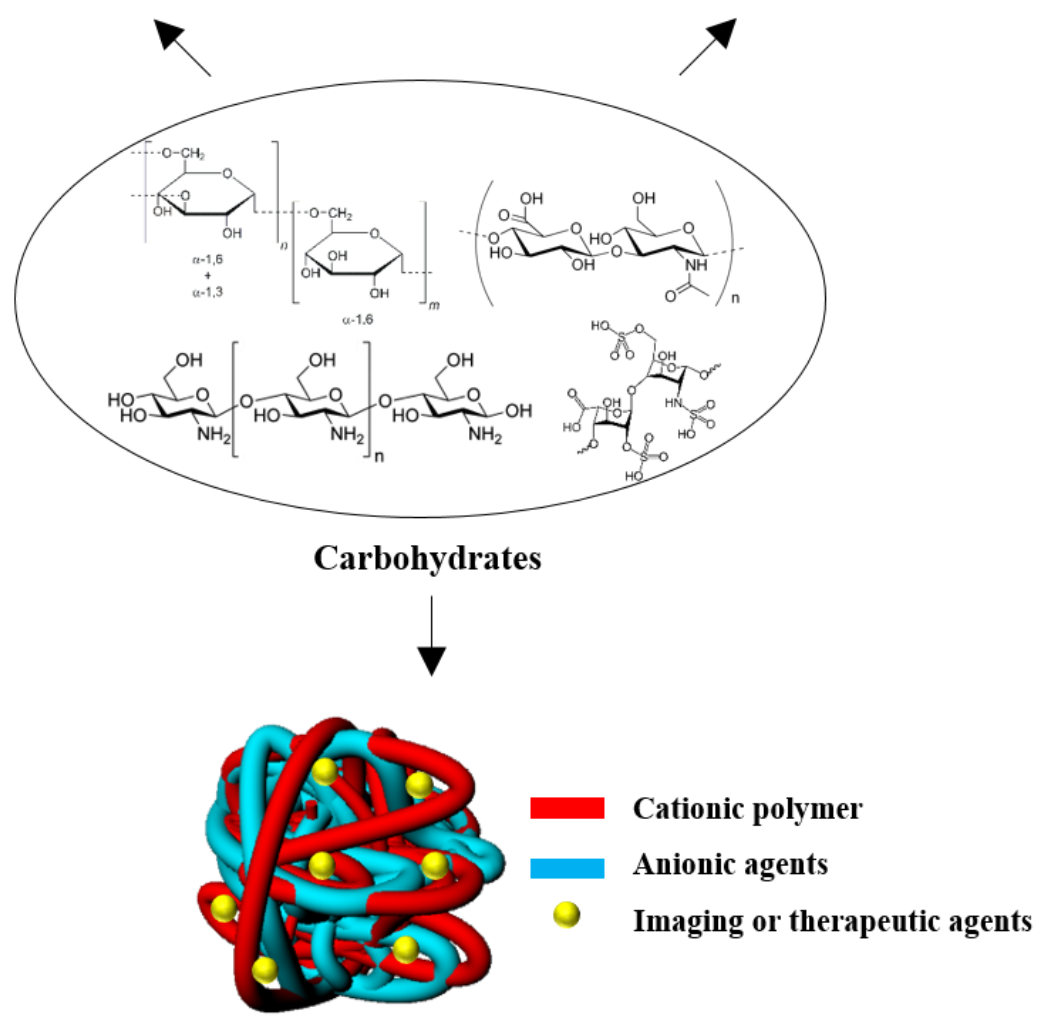

Physically crosslinked nanoparticles

Figure 1. Schematic illustration of representative strategies to prepare carbohydrate-based nanoparticles.

\subsection{Self-Assembled Nanoparticles}

Amphiphilic carbohydrates, capable of forming self-assembled NPs in an aqueous environment, are readily synthesized by covalently attaching the hydrophobic molecules onto the carbohydrate backbone. Such nanoparticles consist of hydrophobic inner cores, surrounded by 
the carbohydrate shell, as the potential reservoir of therapeutic and imaging agents [52-54]. In an attempt to prepare the amphiphilic carbohydrate to form NPs, numerous hydrophobic molecules have been attached at the backbone of polysaccharides, including fatty acids [55], cholesterol [56], bile acids [31, 57], and oligomers [58, 59]. The physicochemical properties of the NPs such as size, surface characteristics, and morphology are primarily dependent on the degree of substitution (DS) of the hydrophobic molecules. In general, the particle size tends to decrease as the DS increases due to the formation of compact inner cores by enhanced hydrophobic interactions. Self-assembled nanoparticles may not form with a low DS value, whereas excess modification of carbohydrates may result in precipitation under aqueous conditions, ascribed to high hydrophobicity. In some cases, the DS significantly affects the biological functions of the parent polysaccharide. For example, excess modification of the carboxylic acid in hyaluronic acid (HA) (> $25 \mathrm{~mol} \%$ ) has decreased the binding affinity to its receptor [60]. Therefore, to develop nanoparticles with the desired physicochemical properties, it is of high importance to find the optimal DS value for each hydrophobic molecule.

In recent years, stimuli-responsive polymeric NPs have been extensively investigated for drug delivery and biomedical imaging [61-65]. Such NPs have been designed to release the payloads by changing their structure in response to endogenous or exogenous stimuli such as changes in $\mathrm{pH}$, enzyme concentrations, temperature, and redox gradients. The stimuli-responsive NPs based on polysaccharides can be prepared by introducing stimuli-labile linkers or hydrophobic moieties into amphiphilic polysaccharides. For example, carboxymethyl dextran derivate was recently synthesized by chemical modification of CMD with lithocholic acid via a bioreducible disulfide linkage which is cleavable at the intracellular environment (CMDSS_LCA, Figure 2) [66]. 


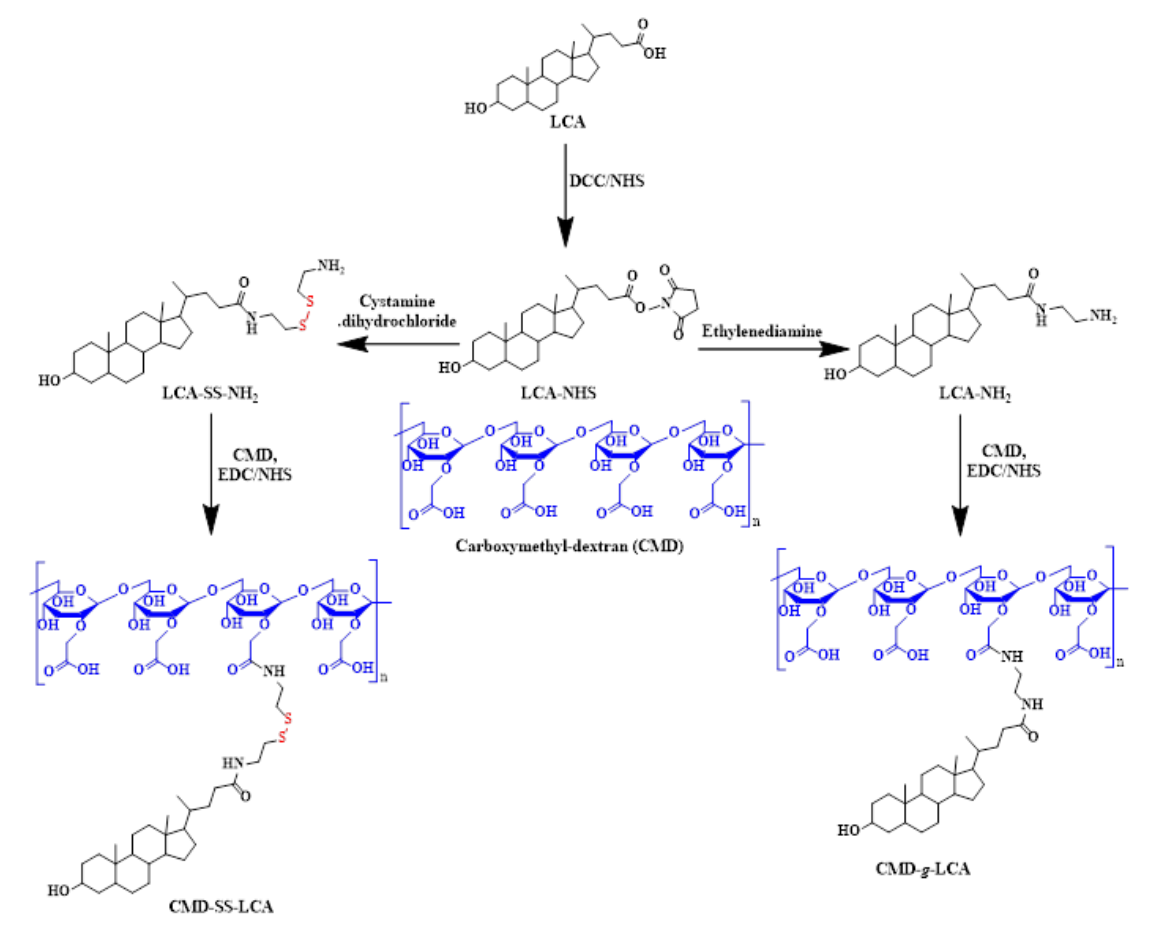

Figure 2. Synthesis of amphiphilic carboxymethyl dextran derivatives with and without the disulfide bond. Adapted with permission from Ref. [66].

Depending on the DS of lithocholic acid, the amphiphilic CMD formed NPs with sizes ranging from 163 to $242 \mathrm{~nm}$. The resulting NPs were labeled with the near-infrared dye, Cy5.5, for nearinfrared fluorescence (NIRF) in vivo imaging, and their core was loaded with the anticancer drug, doxorubicin (DOX) for drug delivery. The CMD-SS-LCA NPs exhibited selective and rapid release of DOX in PBS containing $10 \mathrm{~m} M$ glutathione, a tripeptide that reduces disulfide bonds intracellularly. Using NIRF, the Cy5.5-labeled NPs exhibited preferential distribution at the tumor site after systemic administration. As a consequence, DOX-loaded CMD-SS-LCA NPs exhibited significantly higher antitumor efficacy in vivo, compared to reduction-insensitive CMD NPs. Recently, CMD was also used to prepare hypoxia-responsive, self-assembled NPs with a 2nitroimidazole derivative as the hydrophobic moiety (Figure 3) [49]. Hypoxia is a result of deficient amounts of oxygen reaching tissues, which may occur in many intractable diseases 
including cancer. As seen in Figure 3, the CMD-based NP collects within tumors and delivers drugs at different rates in response to the oxygen levels in the tissue. DOX, loaded within the hydrophobic NP core, can be released in a sustained manner under normoxic conditions (physiological conditions). But in hypoxic conditions, 2-nitroimidazole converts to hydrophilic 2-aminoimidazoles through a series of reductive processes and the release rate of DOX is remarkably increased. When dye-labeled NPs were intravenously injected into the tail vein of the tumor-bearing mice, strong fluorescent signals were detected at the hypoxic region of the tumor site, implying the theranostic potential of NPs.

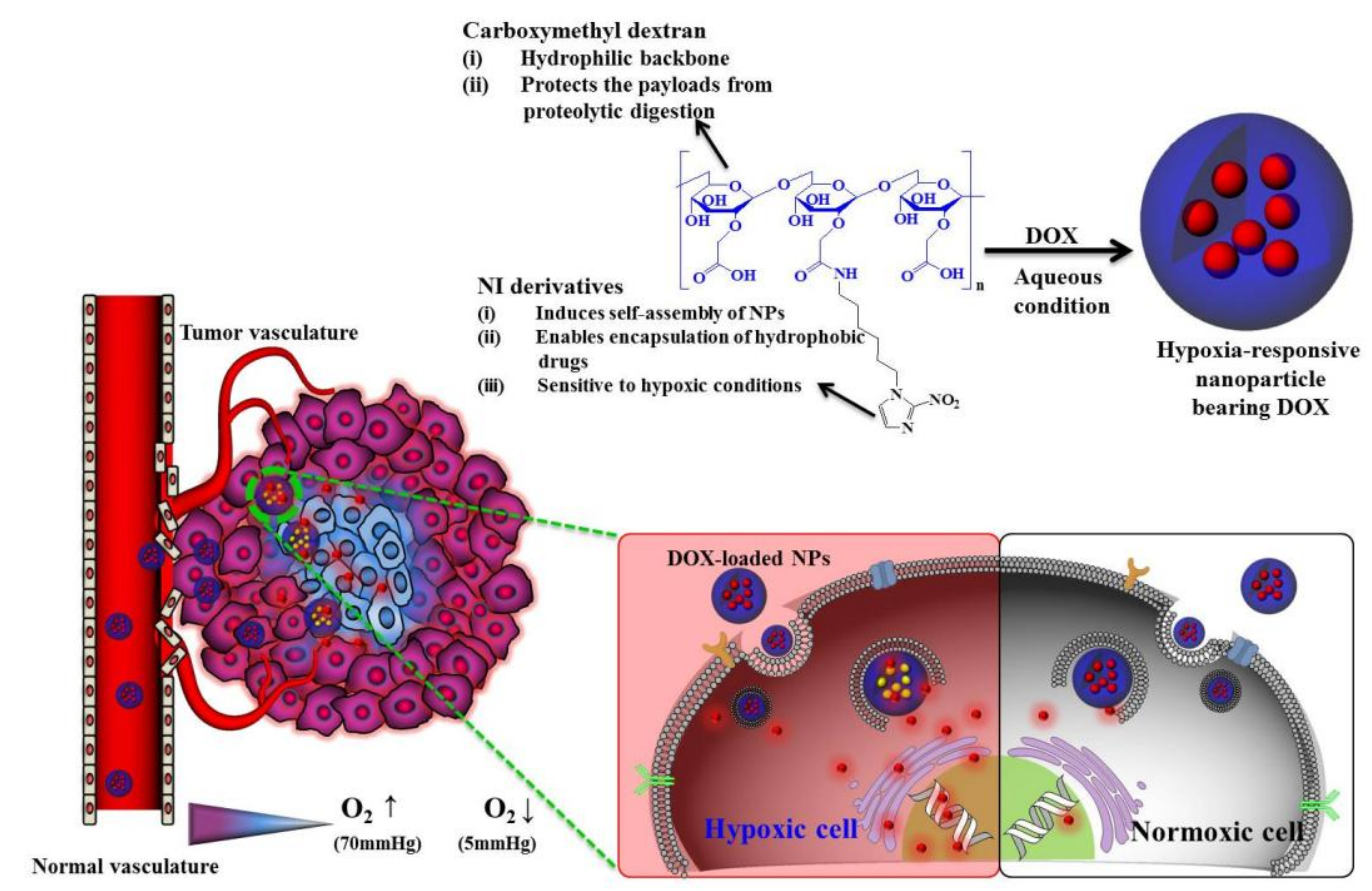

Figure 3. Schematic illustration of the formation of drug-loaded hypoxia-responsive nanoparticles and in vivo tumor-targeting pathways. Adapted with permission from Ref. [49].

In addition to the small molecular compounds, hydrophobic polymers have been conjugated into carbohydrates by numerous approaches [67-69]. The polymer-grafted 
carbohydrates have been extensively prepared through "grafting from" or "grafting to" approaches. In general, the "grafting from" approaches imply synthesis of carbohydrate derivatives by polymerization of monomers from carbohydrate functional groups as initiating species. For example, biodegradable aliphatic polyesters (e.g., polylactide, polycaprolactone, and poly(lactic-co-glycolic acid)) have been grafted onto carbohydrates by ring-opening polymerizations, in which pendant hydroxyl groups in carbohydrates are used as initiating species [70, 71]. The "grafting to" approaches indicate preparation of the amphiphilic carbohydrates based on well-known organic reactions with functionalized polymers such as click-type and condensation reactions [72-75]. The click-type reactions are generally carried out by 1,3-cycloaddition of alkynes and azides in the presence of $\mathrm{Cu}(\mathrm{I})$ complexes, whereas condensation reactions are often performed between carboxylic acids and amino or hydroxyl groups in the presence of carbodiimides. Carbohydrate-based diblock copolymers reacted with lipids or synethic polymers can form NPs, like micelles or polymersomes [48, 76, 77]. In particular, polymersomes have promising potential for theranostics because they can encapsulate both hydrophilic and hydrophobic agents. As a typical example, Schatz et al. prepared a dextranblock-polypeptide copolymer which forms polymersomes mimicking the structure of viruses as a potential carrier of drugs and imaging agents [76]. The block copolymer was simply synthesized by click chemistry using alkyne end-functionalized dextran and azide end-functionalized poly $(\gamma$ benzyl L-glutamate) (PBLG). The same chemistry was also applied to prepare HA-block-PBLG copolymer for tumor-targeted drug delivery and biomedical imaging [78, 79]. This chemistry can be useful to make carbohydrate-based NPs without significant deterioration in the biological functions of parent carbohydrates, securing the significant biological utility of polysaccharides like HA and heparin. 


\subsection{Crosslinked Nanoparticles}

\subsubsection{Ionically Crosslinked Nanoparticles}

Charged polysaccharides can form physically crosslinked NPs in the presence of oppositely charged crosslinkers, which are either small molecules or polymers. Since ionically crosslinked NPs (IC-NPs) are readily prepared in mild conditions without the use of organic solvents at room temperature, they are promising agents to encapsulate fragile drugs (e.g., peptides and proteins) without significant loss of therapeutic function. It should be emphasized that polysaccharidebased IC-NPs are generally considered to be biocompatible and biodegradable, which is an essential requirement for theranostic applications. Among polysaccharides, chitosan has been the most extensively investigated IC-NP, prepared in the presence of the polyvalent anion tripolyphosphate (TPP), as a carrier of small molecular drugs, proteins, and plasmid DNA [8082]. Chitosan has also been used to prepare polyelectrolyte complexes (PECs) by simple complexation with oppositely charged polysaccharides such as HA, alginate, carboxymethyl cellulose, and heparin $[83,84]$. As a negatively charged polysaccharide, alginate can be ionically crosslinked in the presence of bivalent calcium ions. The resulting alginate NPs have been prepared by water-in-oil reverse microemulsion method [85] or ion-induced gelification [86] for the design of drug delivery systems. In some cases, therapeutic proteins and genes, as oppositely charged macromolecules, are directly used to form PECs. For example, Kim et al. prepared PECs based on negatively charged HA and the positively charged protein, poly(ethylene glycol)conjugated tumor necrosis factor-related apoptosis inducing ligand (PEG-TRAIL), as a potential therapeutic strategy for rheumatoid arthritis [87]. The particle size of PECs was dependent on the feed ratio of HA and PEG-TRAIL. The stability of PEG-TRAIL was significantly improved by forming PECs, and sustained release of dye-labeled PEG-TRAIL from PECs was observed using the optical imaging technique after subcutaneous injection into the mouse model. As a 
consequence, PECs exhibited much higher therapeutic efficacy than bare PEG-TRAIL for rheumatoid arthritis.

\subsubsection{Covalently Crosslinked Nanoparticles}

For effective application in theranostics, NPs must possess characteristics to: 1) encapsulate varying amounts of therapeutic and/or imaging agents, 2) exhibit high in vivo stability before reaching the target site, and 3) release the agents at the intended site of action. Among these requirements, the fundamental problem associated with self-assembled NPs is their poor stability in vivo $[88,89]$. In other words, since self-assembled NPs are constructed by weak physical interactions, they often undergo disintegration of the nanoparticular architecture and premature release of the payloads before reaching the target site in vivo. The premature drug release may decrease the therapeutic efficacy and cause undesirable toxicity to normal organs. In recent years, strategies have been proposed to improve NP stability by introducing chemical crosslinkers in NPs [90, 91]. For chemical crosslinking, the reactive functional groups in carbohydrates have been reacted with small molecular or polymeric crosslinkers. The direct chemical crosslinking of bare carbohydrates, however, often produce large NPs with broad size distribution because of inter-particular reactions [69, 92]. In addition, although chemically crosslinked NPs have high colloidal stability, conventional non-cleavable crosslinks within the NPs may act as diffusion barriers that hamper controlled drug release at the target site. To overcome these problems, amphiphilic carbohydrates that can react with cleavable crosslinkers at the intra-molecular level of the self-assembled state need to be prepared.

Introduction of stimuli-labile crosslinkers into the self-assembling NPs is a promising strategy to prepare biostable NPs with narrow size distributions that can release payloads in 
response to stimuli such as low $\mathrm{pH}$ and reductive environments. Based on this concept, Li et al. prepared reversibly stabilized self-assembled NPs by using dextran-lipoic acid (Dex-LA) conjugates [93]. The Dex-LA conjugates could form self-assembled NPs in an aqueous solution and be readily crosslinked by a thiol-disulfide exchange reaction in the presence of dithiothreitol (DTT). Since the disulfide linkage is cleaved at the reductive environment of the intracellular compartments, the resulting NPs can be carriers for intercellular drug delivery and/or diagnostics. Recently, Han et al. prepared bioreducible core-crosslinked particles (CC-NPs) based on HA-block-poly(pyridyl disulfide methacrylate) copolymer (Figure 4) [77]. Compared to non-crosslinked nanoparticles, CC-NPs exhibited higher structural stability in the presence of serum or sodium dodecyl sulfates. Owing to the stimuli-responsive characteristics, the drug was rapidly released from CC-NPs in the presence of glutathione. CC-NPs were also labeled on the surface with a NIRF dye, Cy5.5, to non-invasively monitor the particle in vivo. When systemically administrated into the tumor-bearing mice, the Cy5.5-labeled CC-NPs allowed for clear discrimination of the tumor site by using an optical imaging system. In addition, DOXloaded CC-NPs exhibited excellent antitumor efficacy, demonstrating potential of CC-NPs for theranostic applications. 
(a)
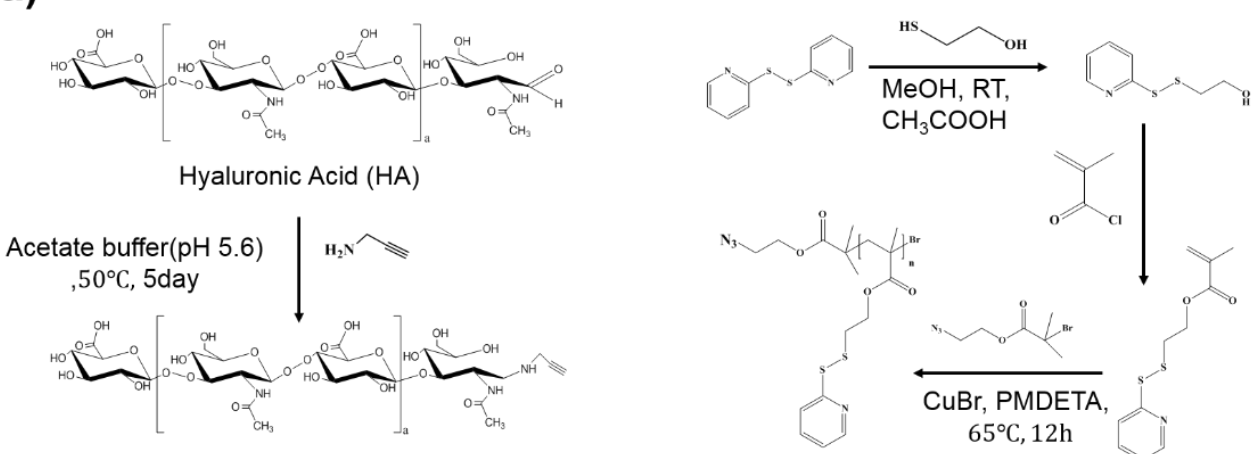

Alkyn-HA
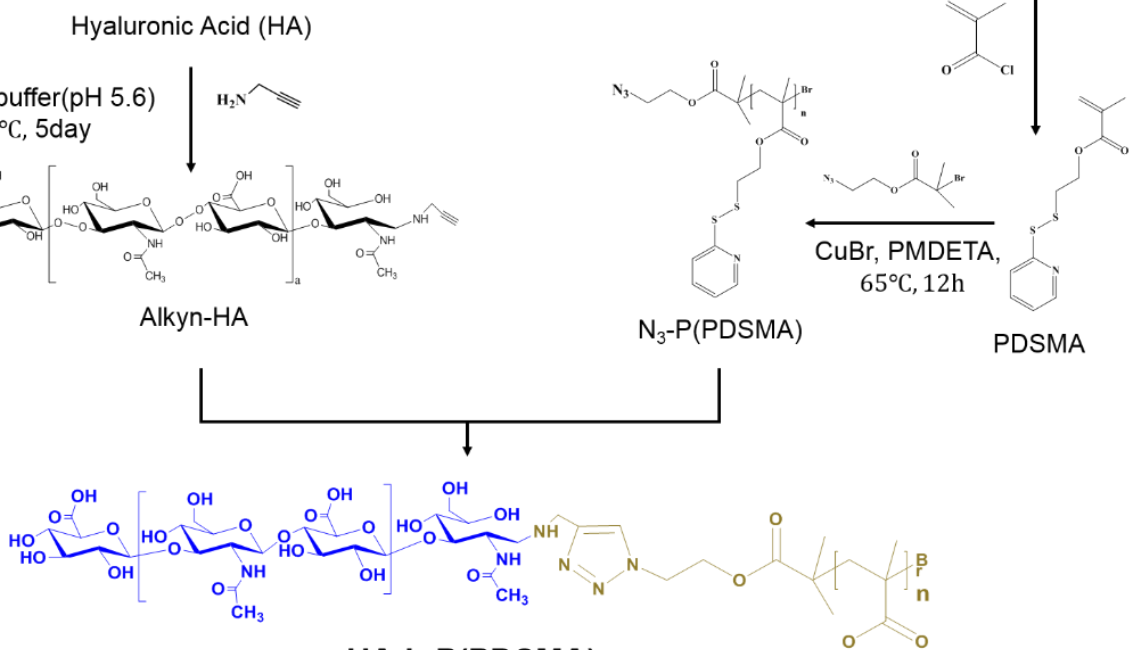

HA-b-P(PDSMA)

(b)

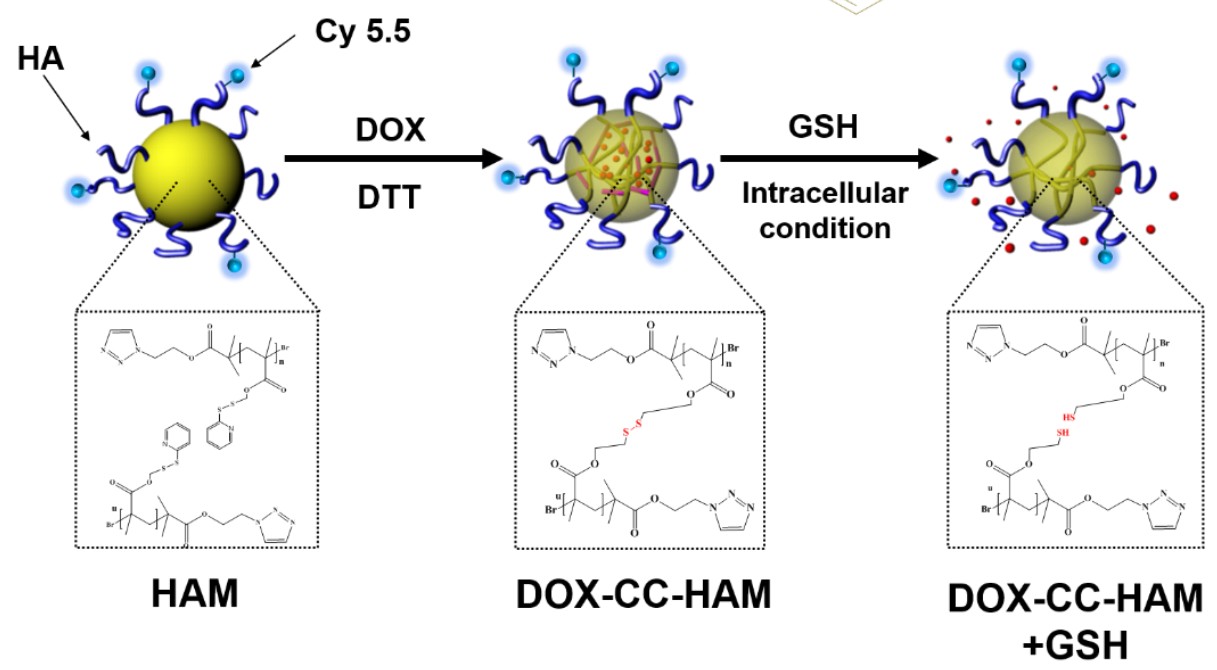

Figure 4. (a) Synthetic scheme of hyaluronic acid (HA)-poly(pyridyl disulfide methacrylate) (P(PDSMA)) conjugate. (b) Schematic illustration of drug release from core-crosslinked hyaluronic acid polymeric micelles. Adapted with permission from Ref. [77]. 
Several strategies have been established to modify carbohydrates towards advantageous NPs - preparing amphiphilic carbohydrate-based materials, forming PECs with oppositely charge materials as well as applying crosslinkers. These techniques set the foundation for carbohydratebased theranostics because they enable multifunctional tasks, including therapeutic delivery and/or molecular imaging. Carbohydrates are abundant and cost-effective molecules, allowing economic scale-up for clinical use. Nonetheless, careful NP characterization on the NP shape, surface charge and chemistry is required to confirm reproducibility during NP manufacturing scale-up. Additionally, when designing theranostic NPs, concentrations of drugs and imaging agents need to be controlled for in vivo delivery. The required dose for a therapeutic may be orders of magnitude different than the amount of signaling agent, depending on the imaging modality. Overall, the imaging and therapeutic approach required for the disease indication may dictate the type of carbohydrate modification used to induce synergy and enable effective theranostic nanomedicine.

\subsection{Stability-Enhancing Strategies}

After systemic administration, self-assembled polysaccharide-based NPs are highly diluted and their structural integrity can be challenged by its dynamic state. Increasing the drug loading content can also destabilize the NP. As discussed in Section 2.2, ionic or covalent cross-linking is an effective method to improve structural stability of polysaccharide-based NPs to increase theranostic functionality in vivo.

Another strategy applied in recent years is mineralization through controlled deposition of inorganic components to develop organic-inorganic hybrid NPs [72, 94, 95]. Calcium phosphate, a major constituent of bone and teeth, has been extensively investigated for mineralization because it is biocompatible and biodegradable [94, 96]. Also, it has been 
demonstrated that biomaterials with anionic functional moieties, like HA, are effectively mineralized by sequential addition of calcium nitrate and ammonium phosphate [72, 94]. Mineralized HA-NPs, loaded with DOX, demonstrate a reduced particle size and slower drug release rate over bare HA-NPs in physiologic conditions ( $\mathrm{pH}$ 7.4) [94]. In slightly acidic conditions, however, the mineralized coat that acted as a diffusion barriers degrades into nontoxic ionic species and the drug release pattern mimics that of bare HA-NPs. Therefore, mineralization of NPs with calcium phosphate can be used to deliver imaging and therapeutic agents with greater stability in physiological conditions than bare NPs, but enhanced delivery in acidic intracellular components like endosomes and lysosomes or in the slightly acidic conditions of the extracellular matrix in tumor regions.

\section{Application of Polysaccharide-Based Nanoparticles for Theranostic Nanomedicine}

Based on the intrinsic properties of polysaccharides matched with the modification techniques set out in Section 2, polysaccharide-based NPs have proven to be useful platforms in nanomedicine (Table 1). Recently, their benefits have warranted the development of multifunctional materials, especially for theranostic nanomedicine. HA and chitosan are two of the most advanced nanoplatforms that have been investigated in theranostics. Therefore, we will introduce their key characteristics and introduce their most recent applications in theranostic nanomedicine from the literature in this section. Table 1 introduces applications of these and additional polysaccharide-based NPs in theranostics since the year 2010, along with the chemical modification applied and the imaging/therapeutic agents used. The majority of nanosystems are adapted for cancer indications and utilize NIRF imaging. As technology advances, other disease indications and imaging modalities (PET/SPECT, MRI, PAI, multimodality) are bound to be addressed. 


\begin{tabular}{|c|c|c|c|c|}
\hline Carbohydrate & $\begin{array}{l}\text { Particle } \\
\text { Enabling } \\
\text { Conjugate } \\
\end{array}$ & Additional Features & Application & References \\
\hline \multirow{28}{*}{ Hyaluronan } & \multirow{6}{*}{$\begin{array}{l}\text { Cholanic } \\
\text { Acid }\end{array}$} & $\mathrm{CuS} \neq, \mathrm{Cy} 5.5^{*}$ & $\begin{array}{l}\text { PAI } \\
\text { PTT } \\
\text { NIRF imaging }\end{array}$ & [97] \\
\hline & & $\mathrm{CPT} \neq, \mathrm{Cy} 5.5^{*}$ & \multirow{2}{*}{$\begin{array}{l}\text { NIRF imaging } \\
\text { Drug delivery }\end{array}$} & {$[98]$} \\
\hline & & IRT $\neq$, Cy5.5* & & [99] \\
\hline & & $\begin{array}{l}\text { Zn(II)-DPA*, Cy5.5*, RNA, } \\
\text { CaP, PTX }{ }^{*}\end{array}$ & $\begin{array}{l}\text { NIRF imaging } \\
\text { Gene delivery } \\
\text { Drug delivery }\end{array}$ & {$[47]$} \\
\hline & & APMA*,Cy5.5*,PEG*, PTX $\ddagger$ & $\begin{array}{l}\text { NIRF imaging } \\
\text { Drug delivery } \\
\text { Improved stability }\end{array}$ & {$[100]$} \\
\hline & & Cy5.5*, FITC* & NIRF imaging for atheroscelerosis & {$[101]$} \\
\hline & \multirow{3}{*}{ Ceramide } & $\mathrm{DOX} \neq, \mathrm{Cy} 5.5^{*}, \mathrm{PEG}^{*}$ & $\begin{array}{l}\text { NIRF imaging } \\
\text { Drug delivery }\end{array}$ & {$[102]$} \\
\hline & & Magnevist $\neq$, DOX $\ddagger$ & $\begin{array}{l}\text { MRI } \\
\text { Drug delivery }\end{array}$ & [103] \\
\hline & & Cy5.5* Gd $^{*}$ & $\begin{array}{l}\text { NIRF imaging } \\
\text { MRI }\end{array}$ & {$[104]$} \\
\hline & pDMAEMA & SiRNA $\neq$, Cy $5.5^{*}$ & $\begin{array}{l}\text { NIRF imaging } \\
\text { Gene delivery }\end{array}$ & [105] \\
\hline & pPDSMA & $\mathrm{Cy} 5.5^{*}, \mathrm{DOX} \neq$ & $\begin{array}{l}\text { NIRF imaging } \\
\text { Drug delivery }\end{array}$ & {$[77]$} \\
\hline & Polypyrrole & $\mathrm{DOX} \neq$ & $\begin{array}{l}\text { Fluorescence activatable imaging } \\
\text { Drug delivery }\end{array}$ & [106] \\
\hline & $\begin{array}{l}\text { Cucurbit[6]ur } \\
\text { il }\end{array}$ & FITC*,FPRL1 peptide spmd* & $\begin{array}{l}\text { Fluorescence imaging } \\
\text { FPRL1 peptide-spmd delivery }\end{array}$ & [107] \\
\hline & Ce6 & & $\begin{array}{l}\text { NIRF imaging } \\
\text { PDT }\end{array}$ & {$[28]$} \\
\hline & PCL & Cy5.5*. DOX $\neq, \mathrm{PDA}^{*}$ & $\begin{array}{l}\text { NIRF imaging } \\
\text { Drug delivery }\end{array}$ & [48] \\
\hline & ICG & PEG* & $\begin{array}{l}\text { NIRF imaging } \\
\text { PAI }\end{array}$ & [108] \\
\hline & SPION & $\mathrm{DOX}^{*}$ & $\begin{array}{l}\text { MRI } \\
\text { Drug delivery }\end{array}$ & [109] \\
\hline & $\begin{array}{l}\text { Sodium } \\
\text { Oleyl }\end{array}$ & SPION $\neq$, PTX $\neq$ & $\begin{array}{l}\text { Fluorescence imaging } \\
\text { MRI }\end{array}$ & [110] \\
\hline & Py & SPION $\neq$ & $\begin{array}{l}\text { MRI } \\
\text { Hydrogel } \\
\text { Drug delivery } \\
\end{array}$ & {$[111]$} \\
\hline & PLGA & DTX $\neq$, coumarin- $6 \neq$, DiR $\ddagger$ & $\begin{array}{l}\text { NIRF imaging } \\
\text { Drug delivery }\end{array}$ & [112] \\
\hline & PVA & DOX $\neq$, TRITC* $*$ & $\begin{array}{l}\text { Fluorescence imaging } \\
\text { Drug delivery }\end{array}$ & [113] \\
\hline & CAEC & rGO $\neq$, DOX $\ddagger$ & NIRF imaging & [114] \\
\hline & SP & $\mathrm{rGO}, \mathrm{DOX} \neq$ & Drug delivery & {$[115]$} \\
\hline & MNCs & SPM & MRI & {$[116]$} \\
\hline & $\mathrm{CB}[6]$ & $\mathrm{DAH}^{*}, \mathrm{SPM}^{*}$ & $\begin{array}{l}\text { NIRF imaging } \\
\text { Hydrogel }\end{array}$ & [117] \\
\hline & $\begin{array}{l}\text { DA } \quad \& \\
\text { AuNCs }\end{array}$ & $\mathrm{DOX} \neq, \mathrm{GFP}$ & $\begin{array}{l}\text { Fluorescence imaging } \\
\text { Drug delivery } \\
\text { PTT }\end{array}$ & {$[118]$} \\
\hline & OVA & & $\begin{array}{l}\text { NIRF Imaging } \\
\text { Foreign antigen delivery }\end{array}$ & {$[119]$} \\
\hline & DLPE,DLPG & MMC $\neq$, FITC $*$ & Adjuvant treatment & {$[120]$} \\
\hline
\end{tabular}




\begin{tabular}{|c|c|c|c|c|}
\hline & & PTX $\ddagger$ & Drug delivery & [121] \\
\hline \multirow{11}{*}{ Dextran } & MA & GNR* & $\begin{array}{l}\text { Fluorescence imaging } \\
\text { PTT }\end{array}$ & [122] \\
\hline & HA, TA & Chondrocytes $\ddagger$ & $\begin{array}{l}\text { Hydrogel } \\
\text { Scaffolds for cartilage tissue engineering }\end{array}$ & [123] \\
\hline & IONPs & VP4*, DOX* & $\begin{array}{l}\text { Cellular MRI and fluorescence imaging } \\
\text { Drug delivery }\end{array}$ & [124] \\
\hline & Lysozyme & $\mathrm{DOX} \ddagger$, GNP $\neq$ & $\begin{array}{l}\text { Fluorescence imaging } \\
\text { Drug delivery } \\
\text { Nanogel }\end{array}$ & {$[32]$} \\
\hline & ICG & PEG* & $\begin{array}{l}\text { NIRF imaging } \\
\text { PTT }\end{array}$ & [125] \\
\hline & SA, DOX & Cisplatin $\neq$ & $\begin{array}{l}\text { Fluorescence imaging } \\
\text { Drug delivery }\end{array}$ & [110] \\
\hline & Ce6 & & $\begin{array}{l}\text { Fluorescence activatable imaging } \\
\text { PDT }\end{array}$ & [30] \\
\hline & NI & DOX $\neq$, Cy $5.5^{*}$ & \multirow{2}{*}{$\begin{array}{l}\text { NIRF imaging } \\
\text { Drug delivery }\end{array}$} & [49] \\
\hline & LCA & DOX $¥$, Cy $5.5^{*}$ & & {$[66]$} \\
\hline & PCL & Cy5.5* & NIRF imaging for rheumatoid arthritis & [126] \\
\hline & PGMA & SPION & MRI for atherosclerosis & [127] \\
\hline \multirow{7}{*}{ Chitosan } & $\mathrm{rGO}$ & DOX $\ddagger$, DNA, IONPs $\ddagger$ & \multirow{2}{*}{$\begin{array}{l}\text { MRI } \\
\text { Drug delivery }\end{array}$} & [128] \\
\hline & IONPs & PEG*, CTX* & & [129] \\
\hline & PheoA & & \multirow{3}{*}{$\begin{array}{l}\text { NIRF imaging } \\
\text { PDT }\end{array}$} & [130] \\
\hline & PpIX & & & {$[46]$} \\
\hline & $\begin{array}{l}\text { Diatrizoic } \\
\text { acid }\end{array}$ & $\mathrm{Ce}^{*}$ & & [131] \\
\hline & GTMAC & PTX $\neq$, siRNA & $\begin{array}{l}\text { NIRF imaging } \\
\text { Gene and drug co-delivery }\end{array}$ & [132] \\
\hline & $\mathrm{CA}$ & DOX $\neq$, siRNA, Cy $5.5^{*}$ & $\begin{array}{l}\text { NIRF imaging } \\
\text { Gene and drug sequential delivery }\end{array}$ & [133] \\
\hline
\end{tabular}




\begin{tabular}{|c|c|c|c|c|}
\hline & $\begin{array}{l}\text { Hydrotropic } \\
\text { VBODENA } \\
\text { oligomers }\end{array}$ & PTX $\neq$, Cy5.5* & $\begin{array}{l}\text { NIRF imaging } \\
\text { Drug delivery }\end{array}$ & {$[58]$} \\
\hline & Psi-Pgp & FITC* ${ }^{*}$, Cy3* ${ }^{*}$, Cy5.5* & \multirow{2}{*}{$\begin{array}{l}\text { NIRF imaging } \\
\text { Gene delivery }\end{array}$} & {$[134]$} \\
\hline & poly-siRNA & FITC*, Cy5.5* & & {$[135]$} \\
\hline \multirow{7}{*}{ Cyclodextrin } & PEI, MSNP & Alexa555, siRNA & $\begin{array}{l}\text { NIRF imaging } \\
\text { Gene delivery }\end{array}$ & {$[136]$} \\
\hline & GNP & PEG*, CUR $\neq$ & $\begin{array}{l}\text { Fluorescence imaging } \\
\text { Drug delivery }\end{array}$ & {$[137]$} \\
\hline & $\begin{array}{l}\text { PDMA, } \\
\text { DOTA-Gd }\end{array}$ & pDNA & $\begin{array}{l}\text { MRI } \\
\text { Gene delivery }\end{array}$ & {$[68]$} \\
\hline & pPTX & FCR-675*, AP-1 peptide* & $\begin{array}{l}\text { NIRF imaging } \\
\text { Drug delivery }\end{array}$ & {$[138]$} \\
\hline & $\begin{array}{l}\text { DOTA-Gd, } \\
\text { PHPMA }\end{array}$ & $\mathrm{DOX}^{*}, \mathrm{FA}^{*}$ & $\begin{array}{l}\text { Fluorescence imaging } \\
\text { MRI }\end{array}$ & {$[124]$} \\
\hline & GNP & $\begin{array}{l}\text { PEG*, Biotin*, } \\
\text { Rhodamin B } \neq\end{array}$ & \multirow{2}{*}{$\begin{array}{l}\text { Fluorescence imaging } \\
\text { Drug delivery }\end{array}$} & {$[139]$} \\
\hline & CMD & $\mathrm{DOX} \ddagger$ & & {$[140]$} \\
\hline \multirow{3}{*}{ Pullulan } & UR & MTX $^{*}$, CA4 $\neq$, FITC* & $\begin{array}{l}\text { Fluorescence imaging } \\
\text { Co-drug delivery }\end{array}$ & {$[141]$} \\
\hline & Doxil ${ }^{\circledR}$ & PEG*, FA*, Cyst* & $\begin{array}{l}\text { Fluorescence imaging } \\
\text { Drug delivery }\end{array}$ & {$[142]$} \\
\hline & PEI, HA & FITC $*$, PTX $\neq$ & $\begin{array}{l}\text { Fluorescence Imaging } \\
\text { Nanogel } \\
\text { Drug delivery }\end{array}$ & {$[143]$} \\
\hline \multirow{3}{*}{ Heparin } & Dendron & $\mathrm{DOX}^{*}$ & $\begin{array}{l}\text { Fluorescence activatable imaging } \\
\text { Drug delivery }\end{array}$ & {$[144]$} \\
\hline & Aminated FA & IR-780キ & $\begin{array}{l}\text { NIRF imaging } \\
\text { PTT }\end{array}$ & {$[145]$} \\
\hline & GNP & PheoA* & $\begin{array}{l}\text { NIRF imaging } \\
\text { PDT }\end{array}$ & {$[29]$} \\
\hline
\end{tabular}

Table 1: Theranostic NP systems based on carbohydrates since 2010.

† loaded, $*$ conjugated Abbreviations: AOT : aerosol-OT; APMA: N-(3-aminopropyl)methacrylamide hydrochloride; AuNCs : gold nanocages; CA: 5-beta-cholanic acid; CA4 : Combretastatin A4; CaP : Calcium phosphate; CAEC : Cholesteryl-2-aminoethylcarbamate; CB[6] : cucurbit[6]uril; Ce6 : Chlorin e6; CMD : Carboxymethyl dextran; CPT : camptothecin; CTX : Chlorotoxin; CUR : Curcumin; Cyst : Cysteamine; DA : dopamine; DAH : 1,6-diaminohexane; DiR : 1,1-Dioctadecyl-3,3,3',3'-tetramethyl indotricarbocyanine iodide; DLPE : 2-Dilauroyl-sn-Glycero-3-Phosphoethanolamine; DLPG : 1,2-Dilauroyl-sn-Glycero-3-Glycerol; DOPA : L3,4-dihydroxyphenylalanine; DOTA : 1,4,7,10-tetraazacyclododecane-1,4,7,10-tetraacetic acid; DOX : doxorubicin; 
DTX : docetaxel; FA : Folic acid; FIONs : ferromagnetic iron oxide nanocubes; GAG : glycosaminoglycan; Gd : Gadolinium; GFP : green fluorescent protein; GNP : Gold nanoparticle; GNR: Gold nanorod; GTMAC : glycidyltrimethylammonium chloride; HA : Hyaluronic acid; ICG : indocyanine green; IONPs : iron oxide nanoparticles; IRT : irinotecan; LCA : lithocholic acid; MA: 3-Mercaptopropionic acid; MHT : Magnetic hyperthermia treatment; MMC : mitomycin C; MNCs : magnetic nano crystals; MSNP: Mesoporous silica nanoparticle; MRI: magnetic resonance imaging; MTX : Methotrexate; NI : 2-nitroimidazole; NIRF: near infrared fluorescence; NPs: nanoparticles; PAI: photoacoustic imaging; PCL : polycaprolactone; PDA: 2-(Pyridyldithio)ethylamine; PDMA : poly(N,N-dimethylaminoethyl methacrylate); pDNA : plasmid DNA; PDT: photodynamic therapy; PEG : Polyethylene glycol; PEI : Polyethylene imine; PGMA : poly(glyclerol methacrylate); PheoA : Pheophorbide a; PHPMA : Polymerzied N-(2-hydroxypropyl) methacrylamide; PpIX: protoporophyrin IX; psi-Pgp : Self-polymerized siRNA targeting Pgp mRNA; pPTX : polymer-PTX conjugate; PTT: photothermal therapy; PTX : Paclitaxel; PVA : poly(vinyl alcohol); Py : Pyrene; rGO : reduced grapheme oxide; SA : succinic anhydride; SPION : superparamagnetic iron oxide nanoparticles; SPM : spermine; SP : spiropyran; TA : Tyramine; UR : Urocanic acid ; Zn(II)DPA: Zn(II)-dipicolyamine

\subsection{Hyaluronic acid-based Theranostic Nanoparticles}

\subsubsection{Advantages of Hyaluronic Acid}

HA, also termed hyaluronan, is a negatively charged, non-sulfated glycosaminoglycan composed of repeating units of D-glucuronic acid and N-acetyl-D-glucosamine bound by betalinkages. HA is found throughout the body in epithelial, connective and neural tissue, especially in the skin, vitreous of the eye and synovial fluid. This polysaccharide plays key roles in cell motility, proliferation and regulates cell-cell adhesion. It is necessary for embryonic development, wound healing repair, and inflammation. Because it is an essential structural molecule, there is approximately $15 \mathrm{~g}$ of $\mathrm{HA}$ in an average $70 \mathrm{~kg}$ person [39]. HA can function as a lubricant and shock absorber, therefore it is FDA approved as an injectable to treat knee pain in osteoarthritis or for facial implants to make skin appear smoother. In its natural state, HA can be found circulating freely or adhering to glycosaminoglycans, cell surface receptors, or proteins through electrostatic interactions or covalent binding. HA binds to its receptors hyaluronatemediated motility (RHAMM), stabilin-2, and CD44, a transmembrane glycoprotein that is known to be over-expressed in many types of cancer [36-38]. When HA is bound to CD44, it is cleaved into lower molecular weight segments as low as $20 \mathrm{kDa}$ ( $\sim 50$ disaccharides units) by the 
membrane anchored enzyme Hyal-2 and readily internalized to the cells [39]. When in the cell, the degraded HA is taken up by endosomes and ultimately lysosomes where it is further digested by Hyal-1. The HA-CD44 targeting has motivated the application of HA as a cancer targeting moiety for drug delivery systems. HA-based NPs (HA-NPs) designed to maintain the targeting and bioadhesive characteristics of unmodified HA have had promising results in theranostic nanomedicine.

\subsubsection{Application of Hyaluronic Acid-Based NPs}

Table 1 introduces recent examples of carbohydrate-based NPs used for theranostic studies. One common approach to form HA-NPs is to conjugate hydrophobic bio-moieties, like cholanic acid [47, 97-101] or ceramide [102-104], to the HA backbone. Then, the amphiphilic conjugate can self-assemble into NPs, as discussed in Section 2. Amphiphilic HA-5 $\beta$-cholanic acid conjugates (HACA) have been examined in head and neck [53, 146], breast [98], and colon [99] cancer animal models. Zhang, et al. developed activatable HACA-NPs labeled with Cy5.5, a NIR dye, and loaded with copper sulfide (HANPC) for tumor-targeted optical/photoacoustic (PA) image-guided photothermal therapy (PTT) [97]. In this theranostic design, either imaging modality indicates where laser irradiation should be applied to initiate PTT; but unlike PA, NIRF imaging allows whole-body image. HANPC acts as an activatable imaging probe that exhibits an enhanced fluorescence signal after the NP degrades at the tumor site. When the NP is intact, the proximity of copper sulfide in the NP core quenches Cy5.5 fluorescence. After the HA backbone of HANPC is degraded by overexpression of Hyal in tumors, Cy5.5 fluorescence is recovered, significantly increasing the fluorescence signal intensity over the healthy tissue. Because copper sulfide is a highly absorbing material, it also serves as a PA imaging and PTT therapeutic agent. PA imaging is an emerging technology that can identify highly absorbing materials at an 
increased depth and improved resolution over fluorescence imaging in vivo. During PTT, the tumor is irradiated with about $808 \mathrm{~nm}$ light and copper sulfide absorbs light energy and induces local heating via the PA effect. Based on these preclinical studies, a tumor inhibition of nearly $90 \%$ was seen in mice treated with the HANPC due to targeted cellular ablation.

Aside from cancer, HACA-NPs show promise in the diagnosis and treatment of artherosclerosis [101]. ApoE-deficient mice fed high cholesterol atherogenic diets demonstrate atherosclerotic plaques that express stabilin-2 and CD44, two targets of HA. Dye-labeled HACA-NPs greatly accumulated in atherosclerotic plaques within the aorta of the diseased animal model, while only minimal fluorescent signal was detected in aortas of healthy animals. When compared with glycol chitosan NPs that penetrate plaques through passive targeting only, HACA-NPs demonstrated nearly twice as much accumulation at the artherosclerotic lesion. As a result, HACA-NPs have potential to non-invasively detect and monitor atherosclerosis and carry therapeutics specifically to vulnerable plaques. With further development of HACA-NPs for clinical translation, theranostic agents may replace currently invasive methods to diagnose plaques like quantitative coronary angiography or intravascular ultrasound and provide targeted treatment for the underlying cause of atherosclerosis by directly suppressing inflammatory mechanisms rather than just alleviating symptoms of the disease.

HA-based NPs can also be functionalized as a non-viral carrier of siRNA, coupled with tumor imaging capabilities [105]. Yoon et al. developed a stimuli-responsive, non-viral carrier of siRNA using HA grafted poly(dimethylaminoethyl methacrylate) (HPD) that can chemically crosslink through disulfide bonds (siRNA-HPD) for gene silencing therapy. Additionally, the siRNA delivery system was designed to respond to two environmental stimuli after NP uptake. First, glutathione, which is nearly 1,000 times more concentrated inside the cell than outside the 
cell, reduces the disulfide bonds that crosslink the NP. Next, intracellular hyaluronidase (Hyal) degrades HA to breakdown the NP and release siRNA within the cell. Although Yoon et al. demonstrate that cross-linking of the siRNA-HPD greatly improved tumor targetability and gene silencing over uncross-linked NPs because of the compact and stable NPs, we stress the important role HA played to improve the drug delivery and imaging application of siRNA-HPD. The polysaccharide allowed CD44 targeting and stealthy receptor-mediated uptake of the drug. Next, its derivable chemical groups were used to label imaging agents on the NP backbone. Finally, because of the intrinsic degradation of HA by Hyal, the siRNA-HPD is able to deliver siRNA directly inside the tumor cell in response to the high Hyal concentration [147]. In another embodiment of a HA-NP based gene carrier, the HA backbone was engineered with an artificial gene binding agent, $\mathrm{Zn}(\mathrm{II})$-DPA and demonstrated effective delivery of siRNA and miRNA for gene silencing or replacement in different types of cancer cells. Choi, et al. demonstrate that HANP coloaded with DOX and the multidrug resistance 1 gene target siRNA can sensitize drugresistant tumor cells and suppress tumor growth in vivo [50]. Although these examples utilize fluorescence imaging to track NP delivery, similar labeling techniques on the HA backbone can be adopted to other imaging agents like radiolabeling for PET/SPECT imaging. Because of its amendable backbone, HA-NPs can serve as a multi-drug carrier and multimodal imaging agent.

As seen in these few examples, HA-NPs are not only useful disease-targeting carriers for imaging and therapy, they are also amendable to different types of nanoformulations and theranostic strategies. With continued investigation of HA-NPs in preclinical and clinical settings, unique solutions to unmet medical needs may be realized. 


\subsubsection{Future of Hyaluronic Acid-Based Nanoparticles for Theranostics}

As preclinical applications of HA-based NPs expand into theranostic nanomedicine, greater scrutiny will be needed for their clinical investigation. The long-term toxicity profile of HA-NPs has not been clearly investigated. Although HA is generally considered nonimmunogenic and biocompatible, studies show that HA found in nature can vary from antiangiogenic, immunosuppressive molecules with sizes at $10^{7} \mathrm{Da}$ to angiogenic, immunestimulatory and inflammatory materials at $20 \mathrm{kDa}$ [39]. Since HA oligomers have distinct biological functions at different molecular weight, does the size or shape of HA-NPs affects its bioactivity in vivo? A well-controlled safety comparison among HA-NP sizes, developed by varying the DS with hydrophobic molecules or molecular weights of HA for example, may identify the range of HA-NPs that can be investigated in clinical trials. Although HA alone can be synthesized with reproducible molecular weight and low polydiversity [154], the development of HA-based NPs will require new characterization techniques and scale-up process development. Importantly, for theranostic applications, imaging and therapeutic activities must be well characterized individually and together.

\subsection{Chitosan-based Theranostic Nanoparticles}

\subsubsection{Advantages of Chitosan}

Chitosan is a positively charged, linear polysaccharide composed of 2-amino-2-deoxyglucopyranose (D-glucosamine) and 2-acetamido-2-deoxy-d-glucopyranose (N-acetyl-Dglucosamine) residues linked through $\beta$-(1-4)-linkages. It is derived by deacetylation of the naturally occurring and abundantly available chitin, a polymer found in the exoskeleton of crustaceans. At least $60 \%$ of chitosan should be made up of glucosamine residues to differ from chitin. Like other polysaccharides, chitosan is biocompatible and mucoadhesive. Depending on 
the degree of deacetylation [148], it is biodegraded in lysosomes [149] into non-toxic amino sugars that are safely absorbed in the body [150]. Importantly, chitosan is amendable to modification like ionic crosslinking and covalent binding to form NPs, nanogels, films, fibers, etc. $[20,21]$. Chitosan is a weak base $\left(\mathrm{pK}_{\mathrm{a}}=6.4\right)$, so in acidic conditions it holds cationic amino groups that can be utilized for polyelectrolyte complexes with metal anions [151] and anionic molecules like citrates or phosphates. But in basic or neutral conditions, chitosan has low water solubility so additional modification with hydrophilic molecules, like glycol [133, 135, 152] or polyethylene glycol (PEG) [153, 154], is required. Then, water soluble chitosan can be chemically conjugated on its primary hydroxyl and amine groups with hydrophobic groups to induce NP self-assembly. Modification of chitosan [155] and advantages of chitosan NPs are thoroughly reviewed elsewhere [20, 21]. Unlike HA-based NPs, chitosan NPs do not display active targeting in vivo, but can have targeting groups conjugated to its backbone to induce receptor-medicated endocytosis. Therefore, chitosan-based NPs can serve as passively or actively targeted, polysaccharide-based NPs in disease animal models. Because chitosan is biocompatible and has numerous derivative possibilities, it is utilized for in vivo theranostic nanomedicine.

\subsubsection{Application of Chitosan-based NPs}

Chitosan is a useful material that upon appropriate NP modification can serve as multifunctional imaging and therapeutic agents. Yoon, et al. utilized two different formulation strategies of modified glycol chitosan-based NPs (GCNPs) to encapsulate doxorubicin (DOX), a hydrophobic chemotherapeutic, (DOX-GCNP) or complex a Bcl-2 siRNA for gene therapy (siRNA-GCNP), while carrying imaging agents on its NP surface (Figure 5) [133]. In this theranostic approach, Yoon, et al. sequentially delivers Bcl-2 siRNA-GCNPs and DOX-GCNPs 
to overcome drug resistance in cancer by down-regulating anti-apoptotic defense mechanisms of cancer cells while triggering apoptosis. DOX administration overtime may induce upregulation of anti-apoptotic proteins, like BCL-2, as a cellular defense mechanism and prevent chemotherapy efficacy. DOX-GCNPs were formulated by chemically conjugating the hydrophobic moiety 5-beta-cholanic acid on the hydrophilic chitosan backbone and DOX was encapsulated to the inner cores of GCNPs by dialysis in a DMSO/water co-solvent environment. On the other hand, siRNA-GCNPs were formulated using thiolated glycol chitosan as the starting material. After about seven mol percent of amine residues of glycol chitosan are modified into thiol groups, thiolated glycol chitosan can self-assemble into NPs via disulfide bonds. Weak charge interactions between negatively charged Bcl-2 self-polymerized siRNA and positively charged chitosan polymer forms a self-assembled nanocomplex. Furthermore, disulfide crosslinking is encouraged in mild buffer between the thiolated glycol chitosan and thiol group at the 5' sense and antisense strands of siRNA. The formation of each NP are depicted in Figure 5A. A similar siRNA delivery strategy was also applied to suppress P-glycoprotein, which is responsible for the efflux of chemotherapeutics in multi-drug resistance, and in vivo results indicated that a combination therapy with sub-therapeutic doses of DOX can effectively inhibit tumor growth in MCF-7/ADR tumor-bearing mice [134]. Despite the different properties of DOX and siRNA, both formulations of GCNPs exhibited similar physiochemical properties and achieved uniform in vivo biodistribution and pharmacokinetics in PC-3 human prostate cancer tumor-bearing mice. Based on in vivo NIRF imaging of Cy5.5-labeled GCNPs loaded with DOX or complexed with siRNA, both drugs accumulated in the tumor vasculature and the cancer cells at the same time. Tumor weight averages in mice models were nearly 9 times lower in the combination group than in the free DOX or DOX-GCNP groups 51 days post-treatment [133]. 
Overall, the combination treatment exhibited effective tumor growth inhibition by suppressing drug resistance mechanisms during chemotherapy. Careful investigation of Bcl-2 expression levels and pharmacokinetic profiles determined the appropriate dosing concentrations, order, and frequency for each GCNP. The use of GCNPs bestowed significantly different types of molecules the same intrinsic physicochemical properties and enabled an engineered treatment strategy. In theranostics, where multiple functions play in concert, using the same drug delivery carrier with expected in vivo behavior for imaging and therapeutic agents can aid in preclinical research as well as in personalizing therapies in the clinic. Generally, theranostic nanomedicine researchers utilize one type of NP to perform multifunctional roles. Here, the authors introduce that sequential delivery may be necessary and it can be controlled by using the same nanoplatform.

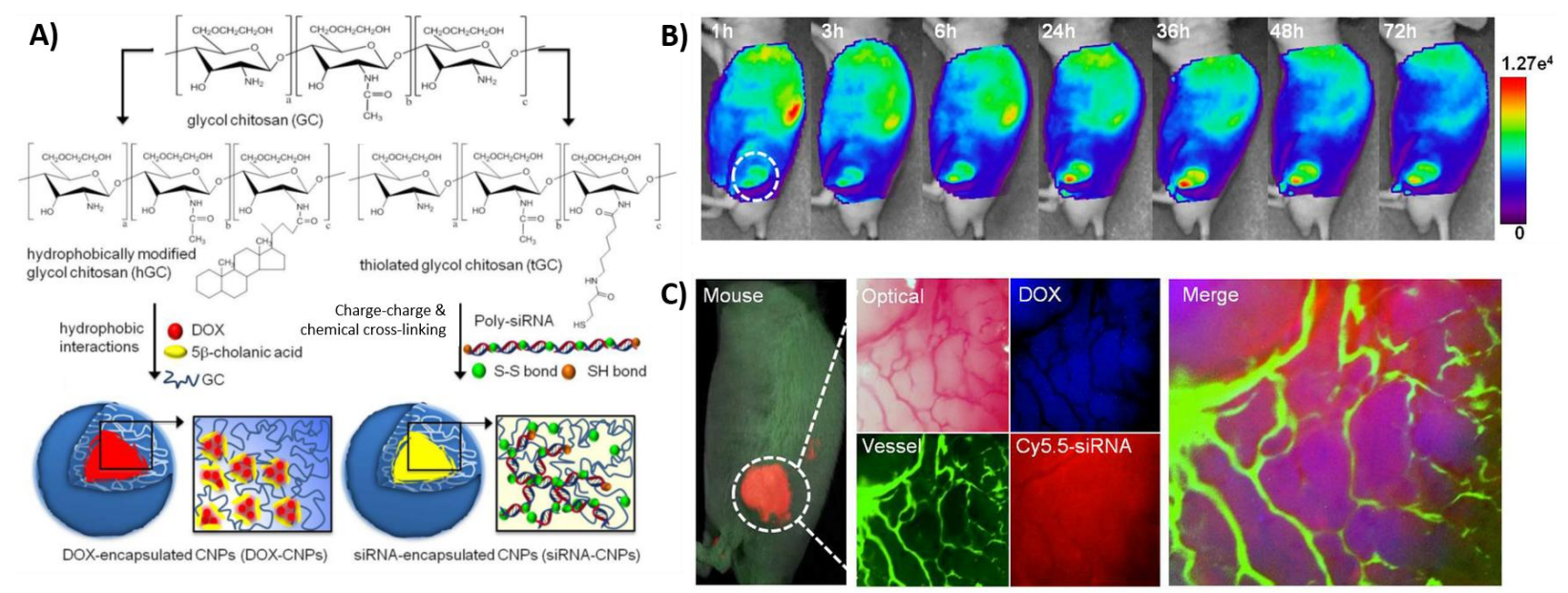

Figure 5: Multifunctional glycol chitosan NPs for NIR fluorescence imaging and drug delivery of hydrophobic drugs and siRNA. A) Nanoparticles formed by modification of glycol chitosan with left) hydrophobic moieties to encapsulate hydrophobic drugs like doxorubicin (DOX) in its core and right) thiol groups to enable cross-linking and charge-charge complexation with negatively charged siRNA. B) Time dependent, whole body distrubtion of dye-labeled glycol 
chitosan NPs by in vivo NIR fluorescence imaging. C) Live imaging of release and localization of both therapeutics from NPs in a solid PC-3 tumor. Adapted with permission from ref. [133].

Chitosan functionalized magnetic graphene (CMG) NPs serve as theranostic platforms for the simultaneous delivery of gene/drugs and superparamagnetic iron oxide (SPIO) NPs for cancer therapy and imaging [128]. Anticancer drugs and genes are delivered to the tumor while SPIO NPs serve as $T_{2}$ contrast agents for noninvasive MRI that is useful for real-time monitoring. The magnetic graphene acts as platform for conjugation of chitosan and its cargo. Based on in vitro and in vivo results, the multifunctional CMG NPs are shown to have effective tumor targeting, gene/drug delivery into tumor cells, $\mathrm{T}_{2}$ contrast for $\mathrm{MR}$ imaging, and biocompatibility [128]. After water-soluble chitosan is covalently bound to carboxylic acid groups on the magnetic graphene via amid bonding, CMG NPs are more soluble and adaptable for in vivo application than the non-functionalized magnetic graphene. Chitosan functionalization allows increased uptake of DOX into cells and therefore enhanced antitumor efficacy compared to free DOX. It also enables pDNA loading on the magnetic graphene, even though chitosan oligomers alone could not deliver nucleic acids to the cells. The results demonstrate the major role chitosan plays to improve theranostic functionality of magnetic graphene. Overall, this study emphasizes that certain nanoplatforms may synergize with the addition of biomaterials like polysaccharides for theranostic applications.

In another embodiment, a chitosan-based theranostic platform was developed to improve intracellular delivery of a DNA repair inhibitor $\mathrm{O}^{6}$-benzylguanine (BG) to glioblastoma multiforme (GBM) cells for chemotherapy sensitization while enabling treatment monitoring by MRI using convection-enhanced delivery (CED) [129]. The system involves multiple 
components: 1) chitosan-grafted-PEG copolymer shell cross-linked through glutathione reducible disulfide linkages, 2) iron oxide core for MR imaging, 3) DNA repair inhibitor to overcome chemotherapy resistance - BG, 4) tumor-targeting peptide - chlorotoxin CTX and 5) NIR dye - Cy5.5 to make NPCP-BG-CTX. CED, based on fluid convection with a pressure gradient during infusion, is used to improve the volume of distribution of the NPs within the brain. In vivo studies in mice bearing orthotopic human primary GMB xenografts demonstrate a large volume of distribution of the NPs in the brain using CED. MR imaging confirm the uptake of the NPs. Because cross-linking can be reduced by intracellular concentrations of glutathione, BG can be released in targeted GBM cells and sensitize the tumor to chemotherapy. The multifunctional and stimuli-response NPCP-BG-CTX delivered with a chemotherapeutic can have a three-fold increase in median survival than untreated animals. Importantly, NP accumulation in the brain after CED could be monitored by MRI and full body NIRF imaging because of the iron oxide core and dye labeling, respectively. Investigation into the long-term stability of these nanoparticles in vivo is required to determine their usefulness in GBM. The preclinical data demonstrate a unique NP system that utilizes numerous drug delivery concepts to improve treatment outcomes. Once again, chitosan was essential to the therapeutic delivery and treatment monitoring properties of the nanosystem.

\subsubsection{Future of Chitosan-Based Nanoparticles for Theranostics}

The key advantages of chitosan-based nanoparticles are its positive charge and reactive functional groups. Chitosan offers a wide range of derivatives that can be leveraged to functionalize imaging and therapeutic agents by chemical conjugation, cross-linking and/or charge-charge interactions. As seen in the above examples, chitosan-based NPs can utilize all of these modification techniques in one theranostic system for simultaneous and/or sequential 
delivery. As a result, chitosan-based NPs have great potential in theranostic nanomedicine. However, unmodified chitosan has poor water solubility in neutral or basic conditions, depending on its degree of deacetylation. Therefore glycol chitosan or PEG-chitosan copolymers are synthesized to fulfill the requirements for in vivo use. As a result, multi-step processing to develop theranostic chitosan-based NPs may be a large deterrent for clinical translation, despite its promising applications in preclinical studies. Solubility and manufacturing issues need to be addressed before chitosan-based theranostics can be applied in humans. Furthermore, in vivo toxicity comparisons among water-soluble chitosan derivatives with reported degrees of deacetylation are needed [156]. Overall, adsorption, distribution, metabolism and exertion (ADME) profiles of well-characterized chitosan-based NPs should be compared.

\section{Perspective}

Polysaccharide-based NPs can have significant advantages in theranostics. They can play an important role in personalized medicine to address clinically unmet needs that are highly variable in patients. Still, most research for polysaccharide-based theranostic NPs is limited to the academic setting. For clinical translation of these potentially beneficial NPs, significant work is required in nano-manufacturing and scale-up, regulatory science that can examine therapy and diagnostics simultaneously, and finally theranostic capabilities in the clinic, like advanced multimodal imaging. Although nanodrugs have been approved by the FDA since 1995 (i.e. Doxil®), next generation engineered and targeted NPs are just entering clinical trials and paving the regulatory path for advanced NPs. For example, BIND-014 from BIND Biosciences is a PEGylated polymer-based NP that carries the anticancer drug DOX and targets the prostate specific membrane antigen (PSMA). It is currently in clinical trials for non-small cell lung cancer (NSCLC) and metastatic castrate-resistant prostate cancer (mCRPC) [34]. Other 
nanodrugs in clinical trials for oncology indications are CRLX101 by Cerulean Pharmaceuticals, Inc. and CALAA-01 by Calando Pharmaceuticals, Inc. These therapeutics are both PEGylated cyclodextrin-based NPs for drug delivery, where CRLX101 carries an anti-cancer drug [157] and CALAA-01 transports siRNA via a targeting moiety [158]. The regulatory standards for these sophisticated NPs and their clinical study results will set precedent for future polysaccharidebased theranostic NPs.

\section{Acknowledgements}

This work was financially supported by the US National Institutes of Health (R00EB013450) and Department of Defense (PC131920) and the Global Research Laboratory Program (NRF2013K1A1A2A02076442), Basic Science Research Program (2012012827) of the NRF. 


\section{References}

[1] N. Adachi, A. Maruyama, T. Ishihara, T. Akaike, Cellular distribution of polymer particles bearing various densities of carbohydrate ligands, J. Biomater. Sci. Polym. Ed., 6 (1994) 463479.

[2] A. Maruyama, T. Ishihara, N. Adachi, T. Akaike, Preparation of nanoparticles bearing high density carbohydrate chains using carbohydrate-carrying polymers as emulsifier, Biomaterials, 15 (1994) 1035-1042.

[3] J. Xie, S. Lee, X. Chen, Nanoparticle-based theranostic agents, Adv. Drug Deliv. Rev., 62 (2010) 1064-1079.

[4] R. Toy, L. Bauer, C. Hoimes, K.B. Ghaghada, E. Karathanasis, Targeted nanotechnology for cancer imaging, Adv. Drug Deliv. Rev., 76 (2014) 79-97.

[5] R. Weissleder, K. Kelly, E.Y. Sun, T. Shtatland, L. Josephson, Cell-specific targeting of nanoparticles by multivalent attachment of small molecules, Nat. Biotechnol., 23 (2005) 14181423.

[6] M. Mammen, S.-K. Choi, G.M. Whitesides, Polyvalent Interactions in Biological Systems: Implications for Design and Use of Multivalent Ligands and Inhibitors, Angew. Chem. Int. Ed., 37 (1998) 2754-2794.

[7] W. Jiang, Y.S. KimBetty, J.T. Rutka, C.W. ChanWarren, Nanoparticle-mediated cellular response is size-dependent, Nat. Nanotechnol., 3 (2008) 145-150.

[8] S.Y. Lee, S.I. Jeon, S. Jung, I.J. Chung, C.-H. Ahn, Targeted multimodal imaging modalities, Adv. Drug Deliv. Rev., 76 (2014) 60-78.

[9] J.V. Jokerst, S.S. Gambhir, Molecular Imaging with Theranostic Nanoparticles, Acc. Chem. Res., 44 (2011) 1050-1060. 
[10] V.P. Chauhan, T. Stylianopoulos, Y. Boucher, R.K. Jain, Delivery of Molecular and Nanoscale Medicine to Tumors: Transport Barriers and Strategies, Annu. Rev. Chem. Biomol. Eng., 2 (2011) 281-298.

[11] R.K. Jain, T. Stylianopoulos, Delivering nanomedicine to solid tumors, Nat. Rev. Clin. Oncol., 7 (2010) 653-664.

[12] H. Maeda, J. Wu, T. Sawa, Y. Matsumura, K. Hori, Tumor vascular permeability and the EPR effect in macromolecular therapeutics: a review, J. Control. Release, 65 (2000) 271-284. [13] H. Maeda, Toward a full understanding of the EPR effect in primary and metastatic tumors as well as issues related to its heterogeneity, Adv. Drug Deliv. Rev., 91 (2015) 3-6.

[14] D. Peer, J.M. Karp, S. Hong, O.C. Farokhzad, R. Margalit, R. Langer, Nanocarriers as an emerging platform for cancer therapy, Nat. Nanotechnol., 2 (2007) 751-760.

[15] L. Brannon-Peppas, J.O. Blanchette, Nanoparticle and targeted systems for cancer therapy, Adv. Drug Deliv. Rev., 56 (2004) 1649-1659.

[16] S.M. Janib, A.S. Moses, J.A. MacKay, Imaging and drug delivery using theranostic nanoparticles, Adv. Drug Deliv. Rev., 62 (2010) 1052-1063.

[17] M.E. Davis, Z. Chen, D.M. Shin, Nanoparticle therapeutics: an emerging treatment modality for cancer, Nat. Rev. Drug Discov., 7 (2008) 771-782.

[18] N. Bertrand, J. Wu, X.Y. Xu, N. Kamaly, O.C. Farokhzad, Cancer nanotechnology: The impact of passive and active targeting in the era of modern cancer biology, Adv. Drug Deliv. Rev., 66 (2014) 2-25.

[19] Z. Liu, Y. Jiao, Y. Wang, C. Zhou, Z. Zhang, Polysaccharides-based nanoparticles as drug delivery systems, Adv. Drug Deliv. Rev., 60 (2008) 1650-1662. 
[20] N. Bhattarai, J. Gunn, M. Zhang, Chitosan-based hydrogels for controlled, localized drug delivery, Adv. Drug Deliv. Rev., 62 (2010) 83-99.

[21] J.H. Park, G. Saravanakumar, K. Kim, I.C. Kwon, Targeted delivery of low molecular drugs using chitosan and its derivatives, Adv. Drug Deliv. Rev., 62 (2010) 28-41.

[22] S. Mizrahy, D. Peer, Polysaccharides as building blocks for nanotherapeutics, Chem. Soc.

Rev., 41 (2012) 2623-2640.

[23] X. Yang, H. Du, J. Liu, G. Zhai, Advanced Nanocarriers Based on Heparin and Its

Derivatives for Cancer Management, Biomacromolecules, 16 (2015) 423-436.

[24] K. Ganguly, K. Chaturvedi, U.A. More, M.N. Nadagouda, T.M. Aminabhavi, Polysaccharide-based micro/nanohydrogels for delivering macromolecular therapeutics, J. Control. Release, 193 (2014) 162-173.

[25] G. Saravanakumar, D.G. Jo, J.H. Park, Polysaccharide-Based Nanoparticles: A Versatile Platform for Drug Delivery and Biomedical Imaging, Curr. Med. Chem., 19 (2012) 3212-3229. [26] D.K. Chatterjee, L.S. Fong, Y. Zhang, Nanoparticles in photodynamic therapy: an emerging paradigm, Adv. Drug Deliv. Rev., 60 (2008) 1627-1637.

[27] J.V. Frangioni, In vivo near-infrared fluorescence imaging, Curr. Opin. Chem. Biol., 7 (2003) 626-634.

[28] H. Kim, Y. Kim, I.-H. Kim, K. Kim, Y. Choi, ROS-Responsive Activatable Photosensitizing Agent for Imaging and Photodynamic Therapy of Activated Macrophages, Theranostics, 4 (2014) 1-11.

[29] L. Li, M. Nurunnabi, M. Nafiujjaman, Y.-k. Lee, K.M. Huh, GSH-mediated photoactivity of pheophorbide a-conjugated heparin/gold nanoparticle for photodynamic therapy, J. Control. Release, 171 (2013) 241-250. 
[30] P. Liu, C. Yue, Z. Sheng, G. Gao, M. Li, H. Yi, C. Zheng, B. Wang, L. Cai, Photosensitizerconjugated redox-responsive dextran theranostic nanoparticles for near-infrared cancer imaging and photodynamic therapy, Polym. Chem., 5 (2014) 874-881.

[31] H.Y. Yoon, H. Koo, K.Y. Choi, S.J. Lee, K. Kim, I.C. Kwon, J.F. Leary, K. Park, S.H. Yuk, J.H. Park, K. Choi, Tumor-targeting hyaluronic acid nanoparticles for photodynamic imaging and therapy, Biomaterials, 33 (2012) 3980-3989.

[32] H. Cai, P. Yao, In situ preparation of gold nanoparticle-loaded lysozyme-dextran nanogels and applications for cell imaging and drug delivery, Nanoscale, 5 (2013) 2892-2900.

[33] M.E. Caldorera-Moore, W.B. Liechty, N.A. Peppas, Responsive Theranostic Systems: Integration of Diagnostic Imaging Agents and Responsive Controlled Release Drug Delivery Carriers, Acc. Chem. Res., 44 (2011) 1061-1070.

[34] J. Hrkach, D. Von Hoff, M.M. Ali, E. Andrianova, J. Auer, T. Campbell, D. De Witt, M. Figa, M. Figueiredo, A. Horhota, S. Low, K. McDonnell, E. Peeke, B. Retnarajan, A. Sabnis, E. Schnipper, J.J. Song, Y.H. Song, J. Summa, D. Tompsett, G. Troiano, T. Van Geen Hoven, J. Wright, P. LoRusso, P.W. Kantoff, N.H. Bander, C. Sweeney, O.C. Farokhzad, R. Langer, S. Zale, Preclinical Development and Clinical Translation of a PSMA-Targeted Docetaxel Nanoparticle with a Differentiated Pharmacological Profile, Sci. Transl. Med., 4 (2012) 128 ra139.

[35] A. Kumari, S.K. Yadav, S.C. Yadav, Biodegradable polymeric nanoparticles based drug delivery systems, Colloids Surf. B., 75 (2010) 1-18.

[36] S.C. Ghosh, S. Neslihan Alpay, J. Klostergaard, CD44: a validated target for improved delivery of cancer therapeutics, Expert Opin. Ther. Targets, 16 (2012) 635-650. 
[37] V. Orian-Rousseau, CD44, a therapeutic target for metastasising tumours, Eur. J. Cancer, 46 (2010) 1271-1277.

[38] H. Ponta, L. Sherman, P.A. Herrlich, CD44: from adhesion molecules to signalling regulators, Nat. Rev. Mol. Cell. Biol., 4 (2003) 33-45.

[39] R. Stern, Hyaluronan catabolism: a new metabolic pathway, Eur. J. Cell. Biol., 83 (2004) 317-325.

[40] M. Sundaram, Y. Qi, Z. Shriver, D. Liu, G. Zhao, G. Venkataraman, R. Langer, R. Sasisekharan, Rational design of low-molecular weight heparins with improved in vivo activity, Proc. Natl. Acad. Sci. U.S.A., 100 (2003) 651-656.

[41] Y.-I. Chung, G. Tae, S. Hong Yuk, A facile method to prepare heparin-functionalized nanoparticles for controlled release of growth factors, Biomaterials, 27 (2006) 2621-2626. [42] G.W. Yip, M. Smollich, M. Götte, Therapeutic value of glycosaminoglycans in cancer, Mol. Cancer Ther., 5 (2006) 2139-2148.

[43] J.H. Park, S. Lee, J.-H. Kim, K. Park, K. Kim, I.C. Kwon, Polymeric nanomedicine for cancer therapy, Prog. Polym. Sci., 33 (2008) 113-137.

[44] R.A. Petros, J.M. DeSimone, Strategies in the design of nanoparticles for therapeutic applications, Nat. Rev. Drug Discov., 9 (2010) 615-627.

[45] J.-W. Yoo, D.J. Irvine, D.E. Discher, S. Mitragotri, Bio-inspired, bioengineered and biomimetic drug delivery carriers, Nat. Rev. Drug Discov., 10 (2011) 521-535.

[46] S.J. Lee, H. Koo, D.-E. Lee, S. Min, S. Lee, X. Chen, Y. Choi, J.F. Leary, K. Park, S.Y. Jeong, I.C. Kwon, K. Kim, K. Choi, Tumor-homing photosensitizer-conjugated glycol chitosan nanoparticles for synchronous photodynamic imaging and therapy based on cellular on/off system, Biomaterials, 32 (2011) 4021-4029. 
[47] K.Y. Choi, O.F. Silvestre, X. Huang, K.H. Min, G.P. Howard, N. Hida, A.J. Jin, N.

Carvajal, S.W. Lee, J.-I. Hong, X. Chen, Versatile RNA Interference Nanoplatform for Systemic Delivery of RNAs, ACS Nano, 8 (2014) 4559-4570.

[48] H.S. Han, T. Thambi, K.Y. Choi, S. Son, H. Ko, M.C. Lee, D.-G. Jo, Y.S. Chae, Y.M.

Kang, J.Y. Lee, J.H. Park, Bioreducible Shell-Cross-Linked Hyaluronic Acid Nanoparticles for Tumor-Targeted Drug Delivery, Biomacromolecules, 16 (2015) 447-456.

[49] J.M. Shin, S.-H. Kim, T. Thambi, D.G. You, J. Jeon, J.O. Lee, B.Y. Chung, D.-G. Jo, J.H. Park, A hyaluronic acid-methotrexate conjugate for targeted therapy of rheumatoid arthritis, Chem. Commun., 50 (2014) 7632-7635.

[50] K.A. Janes, P. Calvo, M.J. Alonso, Polysaccharide colloidal particles as delivery systems for macromolecules, Adv. Drug Deliv. Rev., 47 (2001) 83-97.

[51] C.E. Schanté, G. Zuber, C. Herlin, T.F. Vandamme, Chemical modifications of hyaluronic acid for the synthesis of derivatives for a broad range of biomedical applications, Carbohydr. Polym., 85 (2011) 469-489.

[52] N. Kolishetti, S. Dhar, P.M. Valencia, L.Q. Lin, R. Karnik, S.J. Lippard, R. Langer, O.C. Farokhzad, Engineering of self-assembled nanoparticle platform for precisely controlled combination drug therapy, Proc. Natl. Acad. Sci. U.S.A., 107 (2010) 17939-17944.

[53] K.Y. Choi, H. Chung, K.H. Min, H.Y. Yoon, K. Kim, J.H. Park, I.C. Kwon, S.Y. Jeong, Self-assembled hyaluronic acid nanoparticles for active tumor targeting, Biomaterials, 31 (2010) 106-114.

[54] K.Y. Choi, G. Liu, S. Lee, X. Chen, Theranostic nanoplatforms for simultaneous cancer imaging and therapy: current approaches and future perspectives, Nanoscale, 4 (2012) 330-342. 
[55] Y. Malam, M. Loizidou, A.M. Seifalian, Liposomes and nanoparticles: nanosized vehicles for drug delivery in cancer, Trends Pharmacol. Sci., 30 (2009) 592-599.

[56] Y. Chen, X. Zhu, X. Zhang, B. Liu, L. Huang, Nanoparticles Modified With Tumortargeting scFv Deliver siRNA and miRNA for Cancer Therapy, Mol. Ther., 18 (2010) 16501656.

[57] H.-J. Cho, H.Y. Yoon, H. Koo, S.-H. Ko, J.-S. Shim, J.-H. Lee, K. Kim, I. Chan Kwon, D.D. Kim, Self-assembled nanoparticles based on hyaluronic acid-ceramide (HA-CE) and Pluronic ${ }^{\circledR}$ for tumor-targeted delivery of docetaxel, Biomaterials, 32 (2011) 7181-7190. [58] H. Koo, K.H. Min, S.C. Lee, J.H. Park, K. Park, S.Y. Jeong, K. Choi, I.C. Kwon, K. Kim, Enhanced drug-loading and therapeutic efficacy of hydrotropic oligomer-conjugated glycol chitosan nanoparticles for tumor-targeted paclitaxel delivery, J. Control. Release, 172 (2013) $823-831$.

[59] G. Saravanakumar, K.H. Min, D.S. Min, A.Y. Kim, C.-M. Lee, Y.W. Cho, S.C. Lee, K. Kim, S.Y. Jeong, K. Park, J.H. Park, I.C. Kwon, Hydrotropic oligomer-conjugated glycol chitosan as a carrier of paclitaxel: Synthesis, characterization, and in vivo biodistribution, J. Control. Release, 140 (2009) 210-217.

[60] G. Jiang, K. Park, J. Kim, K.S. Kim, S.K. Hahn, Target Specific Intracellular Delivery of siRNA/PEI-HA Complex by Receptor Mediated Endocytosis, Mol. Pharm., 6 (2009) 727-737. [61] G. Saravanakumar, D.G. Jo, J. H. Park, Polysaccharide-Based Nanoparticles: A Versatile Platform for Drug Delivery and Biomedical Imaging, Curr. Med. Chem., 19 (2012) 3212-3229. [62] T. Thambi, J.H. Park, Recent Advances in Shell-Sheddable Nanoparticles for Cancer Therapy, J. Biomed. Nanotechnol., 10 (2014) 1841-1862. 
[63] S. Mura, J. Nicolas, P. Couvreur, Stimuli-responsive nanocarriers for drug delivery, Nat. Mater., 12 (2013) 991-1003.

[64] M.A.C. Stuart, W.T.S. Huck, J. Genzer, M. Muller, C. Ober, M. Stamm, G.B. Sukhorukov, I. Szleifer, V.V. Tsukruk, M. Urban, F. Winnik, S. Zauscher, I. Luzinov, S. Minko, Emerging applications of stimuli-responsive polymer materials, Nat. Mater., 9 (2010) 101-113.

[65] H. Wei, R.-X. Zhuo, X.-Z. Zhang, Design and development of polymeric micelles with cleavable links for intracellular drug delivery, Prog. Polym. Sci., 38 (2013) 503-535.

[66] T. Thambi, D.G. You, H.S. Han, V.G. Deepagan, S.M. Jeon, Y.D. Suh, K.Y. Choi, K. Kim, I.C. Kwon, G.-R. Yi, J.Y. Lee, D.S. Lee, J.H. Park, Bioreducible Carboxymethyl Dextran Nanoparticles for Tumor-Targeted Drug Delivery, Adv. Healthc. Mater., 3 (2014) 1829-1838. [67] K.L. Kozielski, S.Y. Tzeng, B.A. Hurtado De Mendoza, J.J. Green, Bioreducible Cationic Polymer-Based Nanoparticles for Efficient and Environmentally Triggered Cytoplasmic siRNA Delivery to Primary Human Brain Cancer Cells, ACS Nano, 8 (2014) 3232-3241.

[68] K. Li, B. Liu, Polymer-encapsulated organic nanoparticles for fluorescence and photoacoustic imaging, Chem. Soc. Rev., 43 (2014) 6570-6597.

[69] Y. Wen, J.K. Oh, Recent Strategies to Develop Polysaccharide-Based Nanomaterials for Biomedical Applications, Macromol. Rapid Commun., 35 (2014) 1819-1832.

[70] W. Yuan, J. Yuan, F. Zhang, X. Xie, Syntheses, Characterization, and in Vitro Degradation of Ethyl Cellulose-graft-poly( $\varepsilon$-caprolactone)-block-poly(l-lactide) Copolymers by Sequential Ring-Opening Polymerization, Biomacromolecules, 8 (2007) 1101-1108.

[71] E. Östmark, D. Nyström, E. Malmström, Unimolecular Nanocontainers Prepared by ROP and Subsequent ATRP from Hydroxypropylcellulose, Macromolecules, 41 (2008) 4405-4415. 
[72] Q. He, W. Wu, K. Xiu, Q. Zhang, F. Xu, J. Li, Controlled drug release system based on cyclodextrin-conjugated poly(lactic acid)-b-poly(ethylene glycol) micelles, Int. J. Pharm., 443 (2013) 110-119.

[73] J. Jing, D. Alaimo, E. De Vlieghere, C. Jerome, O. De Wever, B.G. De Geest, R. AuzelyVelty, Tunable self-assembled nanogels composed of well-defined thermoresponsive hyaluronic acid-polymer conjugates, J. Mater. Chem. B., 1 (2013) 3883-3887.

[74] W. Yuan, J. Zhang, H. Zou, T. Shen, J. Ren, Amphiphilic ethyl cellulose brush polymers with mono and dual side chains: Facile synthesis, self-assembly, and tunable temperature-pH responsivities, Polymer, 53 (2012) 956-966.

[75] G.K. Such, A.P.R. Johnston, K. Liang, F. Caruso, Synthesis and functionalization of nanoengineered materials using click chemistry, Prog. Polym. Sci., 37 (2012) 985-1003.

[76] C. Schatz, S. Louguet, J.-F. Le Meins, S. Lecommandoux, Polysaccharide-blockpolypeptide Copolymer Vesicles: Towards Synthetic Viral Capsids, Angew. Chem. Int. Ed., 48 (2009) 2572-2575.

[77] H.S. Han, K.Y. Choi, H. Ko, J. Jeon, G. Saravanakumar, Y.D. Suh, D.S. Lee, J.H. Park, Bioreducible core-crosslinked hyaluronic acid micelle for targeted cancer therapy, J. Control. Release, 200 (2015) 158-166.

[78] K.K. Upadhyay, A.N. Bhatt, E. Castro, A.K. Mishra, K. Chuttani, B.S. Dwarakanath, C. Schatz, J.-F. Le Meins, A. Misra, S. Lecommandoux, In vitro and In vivo Evaluation of Docetaxel Loaded Biodegradable Polymersomes, Macromol. Biosci., 10 (2010) 503-512. [79] K.K. Upadhyay, A.N. Bhatt, A.K. Mishra, B.S. Dwarakanath, S. Jain, C. Schatz, J.-F. Le Meins, A. Farooque, G. Chandraiah, A.K. Jain, A. Misra, S. Lecommandoux, The intracellular 
drug delivery and anti tumor activity of doxorubicin loaded poly( $\gamma$-benzyl l-glutamate)-b-

hyaluronan polymersomes, Biomaterials, 31 (2010) 2882-2892.

[80] N. Csaba, M. Köping-Höggård, M.J. Alonso, Ionically crosslinked

chitosan/tripolyphosphate nanoparticles for oligonucleotide and plasmid DNA delivery, Int. J.

Pharm., 382 (2009) 205-214.

[81] G. Giacalone, H. Hillaireau, P. Capiau, H. Chacun, F. Reynaud, E. Fattal, Stabilization and cellular delivery of chitosan-polyphosphate nanoparticles by incorporation of iron, J. Control. Release, 194 (2014) 211-219.

[82] K.A. Janes, M.P. Fresneau, A. Marazuela, A. Fabra, M.a.J. Alonso, Chitosan nanoparticles as delivery systems for doxorubicin, J. Control. Release, 73 (2001) 255-267.

[83] J.Y. Lee, S.H. Lee, M.H. Oh, J.S. Kim, T.G. Park, Y.S. Nam, Prolonged gene silencing by siRNA/chitosan-g-deoxycholic acid polyplexes loaded within biodegradable polymer nanoparticles, J. Control. Release, 162 (2012) 407-413.

[84] J.H. Hamman, Chitosan Based Polyelectrolyte Complexes as Potential Carrier Materials in Drug Delivery Systems, Mar. Drugs, 8 (2010) 1305-1322.

[85] J.-O. You, C.-A. Peng, Calcium-Alginate Nanoparticles Formed by Reverse Microemulsion as Gene Carriers, Macromol. Symp., 219 (2005) 147-153.

[86] A. Zahoor, S. Sharma, G.K. Khuller, Inhalable alginate nanoparticles as antitubercular drug carriers against experimental tuberculosis, Int. J. Antimicrob. Agents, 26 (2005) 298-303.

[87] Y.-J. Kim, S.Y. Chae, C.-H. Jin, M. Sivasubramanian, S. Son, K.Y. Choi, D.-G. Jo, K. Kim, I. Chan Kwon, K.C. Lee, J.H. Park, Ionic complex systems based on hyaluronic acid and PEGylated TNF-related apoptosis-inducing ligand for treatment of rheumatoid arthritis, Biomaterials, 31 (2010) 9057-9064. 
[88] S.C. Owen, D.P.Y. Chan, M.S. Shoichet, Polymeric micelle stability, Nano Today, 7 (2012) 53-65.

[89] Y.H. Bae, H. Yin, Stability issues of polymeric micelles, J. Control. Release, 131 (2008) 2-

4.

[90] R. Shrestha, M. Elsabahy, S. Florez-Malaver, S. Samarajeewa, K.L. Wooley, Endosomal escape and siRNA delivery with cationic shell crosslinked knedel-like nanoparticles with tunable buffering capacities, Biomaterials, 33 (2012) 8557-8568.

[91] A.N. Koo, H.J. Lee, S.E. Kim, J.H. Chang, C. Park, C. Kim, J.H. Park, S.C. Lee, Disulfidecross-linked PEG-poly(amino acid)s copolymer micelles for glutathione-mediated intracellular drug delivery, Chem. Commun., 48 (2008) 6570-6572.

[92] Y. Sasaki, Y. Tsuchido, S.-i. Sawada, K. Akiyoshi, Construction of protein-crosslinked nanogels with vitamin B6 bearing polysaccharide, Polym. Chem., 2 (2011) 1267-1270.

[93] Y.-L. Li, L. Zhu, Z. Liu, R. Cheng, F. Meng, J.-H. Cui, S.-J. Ji, Z. Zhong, Reversibly Stabilized Multifunctional Dextran Nanoparticles Efficiently Deliver Doxorubicin into the Nuclei of Cancer Cells, Angew. Chem. Int. Ed., 48 (2009) 9914-9918.

[94] S.-Y. Han, H.S. Han, S.C. Lee, Y.M. Kang, I.-S. Kim, J.H. Park, Mineralized hyaluronic acid nanoparticles as a robust drug carrier, J. Mater. Chem., 21 (2011) 7996-8001.

[95] K.K. Perkin, J.L. Turner, K.L. Wooley, S. Mann, Fabrication of Hybrid Nanocapsules by Calcium Phosphate Mineralization of Shell Cross-Linked Polymer Micelles and Nanocages, Nano Letters, 5 (2005) 1457-1461.

[96] H.J. Lee, S.E. Kim, I.K. Kwon, C. Park, C. Kim, J. Yang, S.C. Lee, Spatially mineralized self-assembled polymeric nanocarriers with enhanced robustness and controlled drug-releasing property, Chem. Commun., 46 (2010) 377-379. 
[97] L. Zhang, S. Gao, F. Zhang, K. Yang, Q. Ma, L. Zhu, Activatable Hyaluronic Acid Nanoparticle as a Theranostic Agent for Optical/Photoacoustic Image-Guided Photothermal Therapy, ACS Nano, 8 (2014) 12250-12258.

[98] K.Y. Choi, H.Y. Yoon, J.-H. Kim, S.M. Bae, R.-W. Park, Y.M. Kang, I.-S. Kim, I.C. Kwon, K. Choi, S.Y. Jeong, K. Kim, J.H. Park, Smart Nanocarrier Based on PEGylated Hyaluronic Acid for Cancer Therapy, ACS Nano, 5 (2011) 8591-8599.

[99] K.Y. Choi, E.J. Jeon, H.Y. Yoon, B.S. Lee, J.H. Na, K.H. Min, S.Y. Kim, S.-J. Myung, S. Lee, X. Chen, I.C. Kwon, K. Choi, S.Y. Jeong, K. Kim, J.H. Park, Theranostic nanoparticles based on PEGylated hyaluronic acid for the diagnosis, therapy and monitoring of colon cancer, Biomaterials, 33 (2012) 6186-6193.

[100] H.Y. Yoon, H. Koo, K.Y. Choi, I. Chan Kwon, K. Choi, J.H. Park, K. Kim, Photocrosslinked hyaluronic acid nanoparticles with improved stability for in vivo tumor-targeted drug delivery, Biomaterials, 34 (2013) 5273-5280.

[101] G.Y. Lee, J.-H. Kim, K.Y. Choi, H.Y. Yoon, K. Kim, I.C. Kwon, K. Choi, B.-H. Lee, J.H. Park, I.-S. Kim, Hyaluronic acid nanoparticles for active targeting atherosclerosis, Biomaterials, 53 (2015) 341-348.

[102] H.-J. Cho, I.-S. Yoon, H.Y. Yoon, H. Koo, Y.-J. Jin, S.-H. Ko, J.-S. Shim, K. Kim, I.C. Kwon, D.-D. Kim, Polyethylene glycol-conjugated hyaluronic acid-ceramide self-assembled nanoparticles for targeted delivery of doxorubicin, Biomaterials, 33 (2012) 1190-1200.

[103] J.-H. Park, H.-J. Cho, H.Y. Yoon, I.-S. Yoon, S.-H. Ko, J.-S. Shim, J.-H. Cho, J.H. Park, K. Kim, I.C. Kwon, D.-D. Kim, Hyaluronic acid derivative-coated nanohybrid liposomes for cancer imaging and drug delivery, J. Control. Release, 174 (2014) 98-108. 
[104] H.-J. Cho, H.Y. Yoon, H. Koo, S.-H. Ko, J.-S. Shim, J.-H. Cho, J.H. Park, K. Kim, I.C. Kwon, D.-D. Kim, Hyaluronic acid-ceramide-based optical/MR dual imaging nanoprobe for cancer diagnosis, J. Control. Release, 162 (2012) 111-118.

[105] H.Y. Yoon, H.R. Kim, G. Saravanakumar, R. Heo, S.Y. Chae, W. Um, K. Kim, I.C. Kwon, J.Y. Lee, D.S. Lee, J.C. Park, J.H. Park, Bioreducible hyaluronic acid conjugates as siRNA carrier for tumor targeting, J. Control. Release, 172 (2013) 653-661.

[106] D. Park, Y. Cho, S.-H. Goh, Y. Choi, Hyaluronic acid-polypyrrole nanoparticles as pHresponsive theranostics, Chem. Commun., 50 (2014) 15014-15017. [107] H. Jung, K.M. Park, J.-A. Yang, E.J. Oh, D.-W. Lee, K. Park, S.H. Ryu, S.K. Hahn, K. Kim, Theranostic systems assembled in situ on demand by host-guest chemistry, Biomaterials, $32(2011) 7687-7694$.

[108] K. Miki, T. Inoue, Y. Kobayashi, K. Nakano, H. Matsuoka, F. Yamauchi, T. Yano, K. Ohe, Near-Infrared Dye-Conjugated Amphiphilic Hyaluronic Acid Derivatives as a Dual Contrast Agent for In Vivo Optical and Photoacoustic Tumor Imaging, Biomacromolecules, 16 (2015) 219-227.

[109] M.H. El-Dakdouki, D.C. Zhu, K. El-Boubbou, M. Kamat, J. Chen, W. Li, X. Huang, Development of Multifunctional Hyaluronan-Coated Nanoparticles for Imaging and Drug Delivery to Cancer Cells, Biomacromolecules, 13 (2012) 1144-1151.

[110] D. Šmejkalová, K. Nešporová, G. Huerta-Angeles, J. Syrovátka, D. Jirák, A. Gálisová, V. Velebný, Selective In Vitro Anticancer Effect of Superparamagnetic Iron Oxide Nanoparticles Loaded in Hyaluronan Polymeric Micelles, Biomacromolecules, 15 (2014) 4012-4020. 
[111] Y. Zhang, Y. Sun, X. Yang, J. Hilborn, A. Heerschap, D.A. Ossipov, Injectable In Situ Forming Hybrid Iron Oxide-Hyaluronic Acid Hydrogel for Magnetic Resonance Imaging and Drug Delivery, Macromol. Biosci., 14 (2014) 1249-1259.

[112] J. Huang, H. Zhang, Y. Yu, Y. Chen, D. Wang, G. Zhang, G. Zhou, J. Liu, Z. Sun, D. Sun, Y. Lu, Y. Zhong, Biodegradable self-assembled nanoparticles of poly (d,l-lactide-coglycolide)/hyaluronic acid block copolymers for target delivery of docetaxel to breast cancer, Biomaterials, 35 (2014) 550-566.

[113] S.G. Kupal, B. Cerroni, S.V. Ghugare, E. Chiessi, G. Paradossi, Biointerface Properties of Core-Shell Poly(vinyl alcohol)-hyaluronic Acid Microgels Based on Chemoselective Chemistry, Biomacromolecules, 13 (2012) 3592-3601.

[114] W. Miao, G. Shim, C.M. Kang, S. Lee, Y.S. Choe, H.-G. Choi, Y.-K. Oh, Cholesteryl hyaluronic acid-coated, reduced graphene oxide nanosheets for anti-cancer drug delivery, Biomaterials, 34 (2013) 9638-9647.

[115] A.-A. Nahain, J.-E. Lee, J.H. Jeong, S.Y. Park, Photoresponsive Fluorescent Reduced Graphene Oxide by Spiropyran Conjugated Hyaluronic Acid for in Vivo Imaging and Target Delivery, Biomacromolecules, 14 (2013) 4082-4090.

[116] T. Lee, E.-K. Lim, J. Lee, B. Kang, J. Choi, H.S. Park, J.-S. Suh, Y.-M. Huh, S. Haam, Efficient CD44-targeted magnetic resonance imaging (MRI) of breast cancer cells using hyaluronic acid (HA)-modified MnFe2O4 nanocrystals, Nanoscale Res. Lett., 8 (2013) 149. [117] K.M. Park, J.-A. Yang, H. Jung, J. Yeom, J.S. Park, K.-H. Park, A.S. Hoffman, S.K. Hahn, K. Kim, In Situ Supramolecular Assembly and Modular Modification of Hyaluronic Acid Hydrogels for 3D Cellular Engineering, ACS Nano, 6 (2012) 2960-2968. 
[118] Z. Wang, Z. Chen, Z. Liu, P. Shi, K. Dong, E. Ju, J. Ren, X. Qu, A multi-stimuli responsive gold nanocage-hyaluronic platform for targeted photothermal and chemotherapy, Biomaterials, 35 (2014) 9678-9688.

[119] Y.-H. Lee, H.Y. Yoon, J.M. Shin, G. Saravanakumar, K.H. Noh, K.-H. Song, J.-H. Jeon, D.-W. Kim, K.-M. Lee, K. Kim, I.C. Kwon, J.H. Park, T.W. Kim, A polymeric conjugate foreignizing tumor cells for targeted immunotherapy in vivo, J. Control. Release, 199 (2015) 98105.

[120] G. Bachar, K. Cohen, R. Hod, R. Feinmesser, A. Mizrachi, T. Shpitzer, O. Katz, D. Peer, Hyaluronan-grafted particle clusters loaded with Mitomycin C as selective nanovectors for primary head and neck cancers, Biomaterials, 32 (2011) 4840-4848.

[121] I. Rivkin, K. Cohen, J. Koffler, D. Melikhov, D. Peer, R. Margalit, Paclitaxel-clusters coated with hyaluronan as selective tumor-targeted nanovectors, Biomaterials, 31 (2010) 71067114.

[122] R. Choi, J. Yang, J. Choi, E.-K. Lim, E. Kim, J.-S. Suh, Y.-M. Huh, S. Haam, Thiolated Dextran-Coated Gold Nanorods for Photothermal Ablation of Inflammatory Macrophages, Langmuir, 26 (2010) 17520-17527.

[123] R. Jin, L.S. Moreira Teixeira, P.J. Dijkstra, C.A. van Blitterswijk, M. Karperien, J. Feijen, Enzymatically-crosslinked injectable hydrogels based on biomimetic dextran-hyaluronic acid conjugates for cartilage tissue engineering, Biomaterials, 31 (2010) 3103-3113.

[124] W. Chen, Y. Cao, M. Liu, Q. Zhao, J. Huang, H. Zhang, Z. Deng, J. Dai, D.F. Williams, Z. Zhang, Rotavirus capsid surface protein VP4-coated Fe3O4 nanoparticles as a theranostic platform for cellular imaging and drug delivery, Biomaterials, 33 (2012) 7895-7902. 
[125] P. Liu, C. Yue, B. Shi, G. Gao, M. Li, B. Wang, Y. Ma, L. Cai, Dextran based sensitive theranostic nanoparticles for near-infrared imaging and photothermal therapy in vitro, Chem. Commun., 49 (2013) 6143-6145.

[126] S.-H. Kim, J.-H. Kim, D.G. You, G. Saravanakumar, H.Y. Yoon, K.Y. Choi, T. Thambi, V.G. Deepagan, D.-G. Jo, J.H. Park, Self-assembled dextran sulphate nanoparticles for targeting rheumatoid arthritis, Chem. Commun., 49 (2013) 10349-10351.

[127] D.G. You, G. Saravanakumar, S. Son, H.S. Han, R. Heo, K. Kim, I.C. Kwon, J.Y. Lee, J.H. Park, Dextran sulfate-coated superparamagnetic iron oxide nanoparticles as a contrast agent for atherosclerosis imaging, Carbohyd. Polym., 101 (2014) 1225-1233.

[128] C. Wang, S. Ravi, U.S. Garapati, M. Das, M. Howell, J. Mallela, S. Alwarappan, S.S.

Mohapatra, S. Mohapatra, Multifunctional chitosan magnetic-graphene (CMG) nanoparticles: a theranostic platform for tumor-targeted co-delivery of drugs, genes and MRI contrast agents, J. Mater. Chem. B, 1 (2013) 4396-4405.

[129] Z.R. Stephen, F.M. Kievit, O. Veiseh, P.A. Chiarelli, C. Fang, K. Wang, S.J. Hatzinger, R.G. Ellenbogen, J.R. Silber, M. Zhang, Redox-Responsive Magnetic Nanoparticle for Targeted Convection-Enhanced Delivery of O6-Benzylguanine to Brain Tumors, ACS Nano, 8 (2014) 10383-10395.

[130] I.-h. Oh, H.S. Min, L. Li, T.H. Tran, Y.-k. Lee, I.C. Kwon, K. Choi, K. Kim, K.M. Huh, Cancer cell-specific photoactivity of pheophorbide a-glycol chitosan nanoparticles for photodynamic therapy in tumor-bearing mice, Biomaterials, 34 (2013) 6454-6463.

[131] C.-K. Lim, J. Shin, I.C. Kwon, S.Y. Jeong, S. Kim, Iodinated Photosensitizing Chitosan: Self-Assembly into Tumor-Homing Nanoparticles with Enhanced Singlet Oxygen Generation, Bioconjugate Chem., 23 (2012) 1022-1028. 
[132] W. Wei, P.-P. Lv, X.-M. Chen, Z.-G. Yue, Q. Fu, S.-Y. Liu, H. Yue, G.-H. Ma, Codelivery of mTERT siRNA and paclitaxel by chitosan-based nanoparticles promoted synergistic tumor suppression, Biomaterials, 34 (2013) 3912-3923.

[133] H.Y. Yoon, S. Son, S.J. Lee, D.G. You, J.Y. Yhee, J.H. Park, M. Swierczewska, S. Lee, I.C. Kwon, S.H. Kim, K. Kim, M.G. Pomper, Glycol chitosan nanoparticles as specialized cancer therapeutic vehicles: Sequential delivery of doxorubicin and Bcl-2 siRNA, Sci. Rep., 4 (2014) 6878.

[134] J.Y. Yhee, S. Song, S.J. Lee, S.-G. Park, K.-S. Kim, M.G. Kim, S. Son, H. Koo, I.C. Kwon, J.H. Jeong, S.Y. Jeong, S.H. Kim, K. Kim, Cancer-targeted MDR-1 siRNA delivery using self-cross-linked glycol chitosan nanoparticles to overcome drug resistance, J. Control. Release, 198 (2015) 1-9.

[135] S.J. Lee, M.S. Huh, S.Y. Lee, S. Min, S. Lee, H. Koo, J.-U. Chu, K.E. Lee, H. Jeon, Y. Choi, K. Choi, Y. Byun, S.Y. Jeong, K. Park, K. Kim, I.C. Kwon, Tumor-Homing PolysiRNA/Glycol Chitosan Self-Cross-Linked Nanoparticles for Systemic siRNA Delivery in Cancer Treatment, Angew. Chemie Int. Ed., 51 (2012) 7203-7207.

[136] J. Shen, H.-C. Kim, H. Su, F. Wang, J. Wolfram, D. Kirui, J. Mai, C. Mu, L.-N. Ji, Z.-W. Mao, H. Shen, Cyclodextrin and Polyethylenimine Functionalized Mesoporous Silica Nanoparticles for Delivery of siRNA Cancer Therapeutics, Theranostics, 4 (2014) 487-497. [137] D.N. Heo, W.-K. Ko, H.-J. Moon, H.-J. Kim, S.J. Lee, J.B. Lee, M.S. Bae, J.-K. Yi, Y.-S. Hwang, J.B. Bang, E.-C. Kim, S.H. Do, I.K. Kwon, Inhibition of Osteoclast Differentiation by Gold Nanoparticles Functionalized with Cyclodextrin Curcumin Complexes, ACS Nano, 8 (2014) 12049-12062. 
[138] R. Namgung, Y. Mi Lee, J. Kim, Y. Jang, B.-H. Lee, I.-S. Kim, P. Sokkar, Y.M. Rhee, A.S. Hoffman, W.J. Kim, Poly-cyclodextrin and poly-paclitaxel nano-assembly for anticancer therapy, Nat. Commun., 5 (2014) 3702.

[139] D.N. Heo, D.H. Yang, H.-J. Moon, J.B. Lee, M.S. Bae, S.C. Lee, W.J. Lee, I.-C. Sun, I.K. Kwon, Gold nanoparticles surface-functionalized with paclitaxel drug and biotin receptor as theranostic agents for cancer therapy, Biomaterials, 33 (2012) 856-866.

[140] M. Sivasubramanian, T. Thambi, V.G. Deepagan, G. Saravanakumar, H. Ko, Y.M. Kang, J.H. Park, Carboxymethyl Dextran-Cyclodextrin Conjugate as the Carrier of Doxorubicin, J. Nanosci. Nanotechnol., 13 (2013) 7271-7278.

[141] Y. Wang, H. Chen, Y. Liu, J. Wu, P. Zhou, Y. Wang, R. Li, X. Yang, N. Zhang, pHsensitive pullulan-based nanoparticle carrier of methotrexate and combretastatin A4 for the combination therapy against hepatocellular carcinoma, Biomaterials, 34 (2013) 7181-7190. [142] A. Scomparin, S. Salmaso, A. Eldar-Boock, D. Ben-Shushan, S. Ferber, G. Tiram, H. Shmeeda, N. Landa-Rouben, J. Leor, P. Caliceti, A. Gabizon, R. Satchi-Fainaro, A comparative study of folate receptor-targeted doxorubicin delivery systems: Dosing regimens and therapeutic index, J. Control. Release, 208 (2015) 106-120.

[143] H. Yim, S.-j. Park, Y.H. Bae, K. Na, Biodegradable cationic nanoparticles loaded with an anticancer drug for deep penetration of heterogeneous tumours, Biomaterials, 34 (2013) 76747682.

[144] W. She, N. Li, K. Luo, C. Guo, G. Wang, Y. Geng, Z. Gu, Dendronized heparin-doxorubicin conjugate based nanoparticle as $\mathrm{pH}$-responsive drug delivery system for cancer therapy, Biomaterials, 34 (2013) 2252-2264. 
[145] C. Yue, P. Liu, M. Zheng, P. Zhao, Y. Wang, Y. Ma, L. Cai, IR-780 dye loaded tumor targeting theranostic nanoparticles for NIR imaging and photothermal therapy, Biomaterials, 34 (2013) 6853-6861.

[146] H.S. Han, J. Lee, H.R. Kim, S.Y. Chae, M. Kim, G. Saravanakumar, H.Y. Yoon, D.G. You, H. Ko, K. Kim, I.C. Kwon, J.C. Park, J.H. Park, Robust PEGylated hyaluronic acid nanoparticles as the carrier of doxorubicin: Mineralization and its effect on tumor targetability in vivo, J. Control. Release, 168 (2013) 105-114.

[147] V.B. Lokeshwar, B.L. Lokeshwar, H.T. Pham, N.L. Block, Association of Elevated Levels of Hyaluronidase, a Matrix-degrading Enzyme, with Prostate Cancer Progression, Cancer Res., 56 (1996) 651-657.

[148] K. Tomihata, Y. Ikada, In vitro and in vivo degradation of films of chitin and its deacetylated derivatives, Biomaterials, 18 (1997) 567-575.

[149] K.Y. Lee, W.S. Ha, W.H. Park, Blood compatibility and biodegradability of partially Nacylated chitosan derivatives, Biomaterials, 16 (1995) 1211-1216.

[150] T. Kean, M. Thanou, Biodegradation, biodistribution and toxicity of chitosan, Adv. Drug Deliv. Rev., 62 (2010) 3-11.

[151] E. Guibal, T. Vincent, R. Navarro, Metal ion biosorption on chitosan for the synthesis of advanced materials, J. Mater. Sci., 49 (2014) 5505-5518.

[152] J.H. Na, H. Koo, S. Lee, K.H. Min, K. Park, H. Yoo, S.H. Lee, J.H. Park, I.C. Kwon, S.Y. Jeong, K. Kim, Real-time and non-invasive optical imaging of tumor-targeting glycol chitosan nanoparticles in various tumor models, Biomaterials, 32 (2011) 5252-5261. 
[153] N. Bhattarai, H.R. Ramay, J. Gunn, F.A. Matsen, M. Zhang, PEG-grafted chitosan as an injectable thermosensitive hydrogel for sustained protein release, J. Control. Release, 103 (2005) 609-624.

[154] O. Germershaus, S.R. Mao, J. Sitterberg, U. Bakowsky, T. Kissel, Gene delivery using chitosan, trimethyl chitosan or polyethylenglycol-graft-trimethyl chitosan block copolymers: Establishment of structure-activity relationships in vitro, J. Control. Release, 125 (2008) 145154.

[155] M.N.V.R. Kumar, R.A.A. Muzzarelli, C. Muzzarelli, H. Sashiwa, A.J. Domb, Chitosan Chemistry and Pharmaceutical Perspectives, Chem. Rev., 104 (2004) 6017-6084.

[156] S.C. Tan, E. Khor, T.K. Tan, S.M. Wong, The degree of deacetylation of chitosan: advocating the first derivative UV-spectrophotometry method of determination, Talanta, 45 (1998) 713-719.

[157] S. Svenson, M. Wolfgang, J. Hwang, J. Ryan, S. Eliasof, Preclinical to clinical development of the novel camptothecin nanopharmaceutical CRLX101, J. Control. Release, 153 (2011) 49-55.

[158] J.E. Zuckerman, I. Gritli, A. Tolcher, J.D. Heidel, D. Lim, R. Morgan, B. Chmielowski, A. Ribas, M.E. Davis, Y. Yen, Correlating animal and human phase Ia/Ib clinical data with CALAA-01, a targeted, polymer-based nanoparticle containing siRNA, Proc. Natl. Acad. Sci. U.S.A., 111 (2014) 11449-11454. 